\title{
High speed plasma diagnostics for laser plasma interaction and fusion studies
}

\author{
V N RAI, M SHUKLA, H C PANT and D D BHAWALKAR \\ Laser Programme, Centre for Advanced Technology, PO CAT, Indore 452013, \\ India \\ e-mail: vnrai@cat.ernet.in
}

MS received 26 February 1998; revised 25 May 1999

\begin{abstract}
Laser plasma interaction and fusion studies involve many high speed plasma diagnostics to determine the various parameters for explaining the physical processes taking place in plasma. Detection and analysis of short-term or transient radiations (X-ray and visible) are the bases for diagnosing the physical processes occurring during laser-plasma interaction or similar radiation-emitting processes. This paper reviews the development of various high speed plasma diagnostics which are not only applicable in determining the temporal, spatial and spectral properties of X-rays for this purpose but also have wide use in various other fields of research.
\end{abstract}

Keywords. Laser-plasma interaction; fusion studies; transient radiations; high speed plasma.

\section{Introduction}

Interaction of a high intensity laser light with matter (solid target) first vaporizes material from the surface which ultimately gets converted into plasma (ionized gas). This laser produced plasma expands into the vacuum where propagation of laser light is modified by the free electrons present in the plasma (Jacob et al 1976; Cairn \& Sanderson 1980; Max 1982; Kruer 1988; Radziemski \& Cramers 1989). The plasma formed by a high intensity or small time duration laser has very steep density and temperature gradients as compared to the plasma formed by the low intensity or long time duration laser. The density gradient in this region plays a very important role in the mechanism of light absorption and in the partition of absorbed energy between thermal and non-thermal particle distribution. There are three basic mechanisms through which intense laser light may interact with plasma (Kruer 1988). The first mechanism is simple electron-ion collision, or inverse bremsstrahlung, where the electric field of the incident light agitates the electrons, which then lose this energy in classical collision with ions. This mechanism is important with shallow density gradients. Parametric processes also take part most efficiently when the gradient is shallow. These are the processes in which intense laser field drives one or more longitudinal waves out of the noise in three-wave parametric 
interaction. There are parametric decay processes where laser light decays into a high frequency electron acoustic wave and a low frequency ion acoustic wave, conserving energy and momentum. An important short pulse absorption mechanism is the resonance absorption. When plane-polarized light is obliquely incident on a plasma surface, a radial component of the electric field resonates with plasma frequency and causes large transfer of energy to electrons near critical density $\left(n_{c}\right)$ surface in the plasma. Critical density for a given laser wavelength is given by,

$$
n_{c}=10^{21} / \lambda^{2} \mathrm{~cm}^{-3}
$$

where $\lambda$ is in microns. Energy absorbed at or below the critical density is then conducted towards the ablation surface by various transport processes. As a result of laser-plasma interaction, various processes take place such as ablation of material, target acceleration, high energy particle emission, and generation of various parametric instabilities, as well as emission of radiations ranging from the visible to hard X-rays (Radziemski \& Cramers 1989). Major physical issues that need to be addressed (Nakai 1990; Hooper \& Osborne 1995 ) during laser plasma interaction studies in laser-produced plasma and inertial confinement fusion experiments involve three major areas of study. The first one is the study of energy coupling to the target, which has many sub-areas such as laser light absorption, nonlinear interaction, electron energy transport, ablation of material from target and preheating of target, as well as radiation conversion and confinement. The second important field of interest is the study of implosion symmetry and stability of the targets, particularly in the case of microballoon experiments. This involves topics such as irradiation uniformity, Raleigh-Taylor (R-T) instability at the ablation front of the target and R-T instability at the stagnation phase, as well as the generation of shocks. The third and most important field of interest, from the fusion point of view, is the investigation of ignition and fusion burn, which involves the study of fusion product and burn history during inertial confinement fusion. Another very important area of investigation is the computer simulation of all the physical processes mentioned above. The study of the above physical processes in the laboratory needs various types of high-speed diagnostics. It has been noticed that X-rays are emitted at all stages of the absorption, interaction and transport regime. At densities near and slightly above critical, $\sim 70 \%$ of the incident laser energy may be re-emitted as $\mathrm{X}$-rays with energy ranging from $50 \mathrm{eV}$ to $1 \mathrm{keV}$ or above, depending on the temperature of the plasma. The X-rays emitted produce line as well as continuum spectra. As stated earlier, along with X-rays, visible as well as ultraviolet light is also emitted, which makes the laser-produced plasma an intense source of radiation in the spectral range from the visible to X-rays of energy over $1 \mathrm{keV}$ or even several tens of keV. Generally, plasma lasts for a few tens of picoseconds to a few tens of nanoseconds depending on the duration of the laser beam. Study of X-ray emission from plasma can be helpful in many ways. An intense small-size and short-time duration X-ray source has many applications in various fields of research, particularly in laser-produced plasma and inertial confinement fusion as diagnostic aids. It can provide information about the physical processes taking place in plasma. Study of self-emitted X-rays from laser-irradiated target and X-ray backlighting of the source play an important role in experiments with planar targets as well as in inertial confinement fusion experiments to investigate all the physics aspects as discussed above and particularly hydrodynamics and compression of planar or spherical microballoon targets (Jacob et al 1976; Attwood 1978; Sigel et al 1984). The X-rays emitted from the plasma have a wide spectral range which can be divided into three categories as XUV $(10-100 \mathrm{eV}$ or $1200-120 \AA)$, soft X-rays $(100-1000 \mathrm{eV}$ or 
120-12 $\AA$ ) and hard X-rays (above $1 \mathrm{KeV}$ or below $12 \AA$ ). Various types of X-ray and optical diagnostics are needed to determine the temporal, spectral and spatial characteristics (Jacob et al 1976; Attwood 1978; Stradling et al 1983; Sigel et al 1984; Alexandrov et al 1988; Tsakiris 1989) of radiation being emitted from laser-produced plasma with better resolution. Many advanced diagnostics, such as vacuum photodiode, $\mathrm{X}$ ray and optical streak camera, X-ray pin-hole camera, transmission grating spectrograph, polyvinyledene diflouride (PVDF) sensor and optical shadowgraphy, have been developed in our laboratory.

The main aim of this paper is to review briefly these important high-speed diagnostics for laser-produced plasma and inertial confinement fusion experiments, which are not available commercially.

\section{X-ray vacuum photodiode}

Vacuum photodiode (Beck 1976; Adams et al 1987; Armentraut et al 1988; Lee et al 1988) is a simple but important diagnostic technique for X-ray emission studies in laser-produced plasma and fusion experiments [Inertial Confinement Fusion (ICF) or Magnetic Confinement Fusion (MCF)]. It works on the principle of the photoelectric effect where electrons emitted from the photocathode under the action of UV or X-rays are collected by the anode biased at positive potential. The electric current $i(t)$ flowing in the circuit generates a potential drop $v(t)$ across the resistance $R$ such that $v(t)=R i(t)$. This voltage is proportional to the radiation flux and is displayed on the oscilloscope. Nowadays various $\mathrm{X}$-ray detectors available, such as semiconductor diodes and surface barrier detectors. suffer from saturation problems, whereas other high speed X-ray detectors, such as Xray streak cameras and framing cameras, have some advantages in time-resolution measurements but involve complicated electronic circuitry. Many vacuum photodiodes have been reported in literature with time response varying from 60 to $450 \mathrm{ps}$ (Beck 1976; Adams et al 1987; Armentraut et al 1988) but of complicated design. Some singleand four-channel X-ray vacuum photodiodes have been developed (Rai et al 1995b; Shukla et al 1997a) where each photodiode has $50 \Omega$ coaxial transmission line geometry. It consists of a mesh, which acts as the anode while a circular metal disc behind the mesh acts as the photocathode. Signals from the photocathode are recorded on the fast oscilloscope across $50 \mathrm{ohm}$ termination. The $50 \Omega$ coaxial geometry as well as the transit time of electrons from cathode to anode ensures fast detection of short duration X-rays by the system. The photodiodes are windowless and the materials used are ultrahigh vacuumcompatible, except teflon when used as insulator material. However, replacement of teflon with ceramic insulation and of viton o-rings seals with indium wire or copper gasket seals makes the system perfectly compatible for ultrahigh vacuum. This however is not required for the present set of experiments where the vacuum ranges from $10^{-2}-10^{-6}$ torr $(1$ torr $=133 \mathrm{~Pa}$ ). The system is fixed inside the chamber through a port such that X-rays directly fall on the photocathode. It can provide time resolutions of $\approx 100 \mathrm{ps}$ if used with a fast oscilloscope of $1 \mathrm{GHz}$ bandwidth. This detector remains insensitive to neutrons and $\gamma$-ray flux (Armentrout et al 1988; Lee et al 1988; catalogue of vacuum photodiode from KMS Fusion Inc., USA). which makes it suitable for use in fusion-related experiments, MCF and ICF. In general. this detector provides temporal evolution of X-rays. However, spectral information is also obtained from these photodiodes by using suitable filters in front of the detectors. 


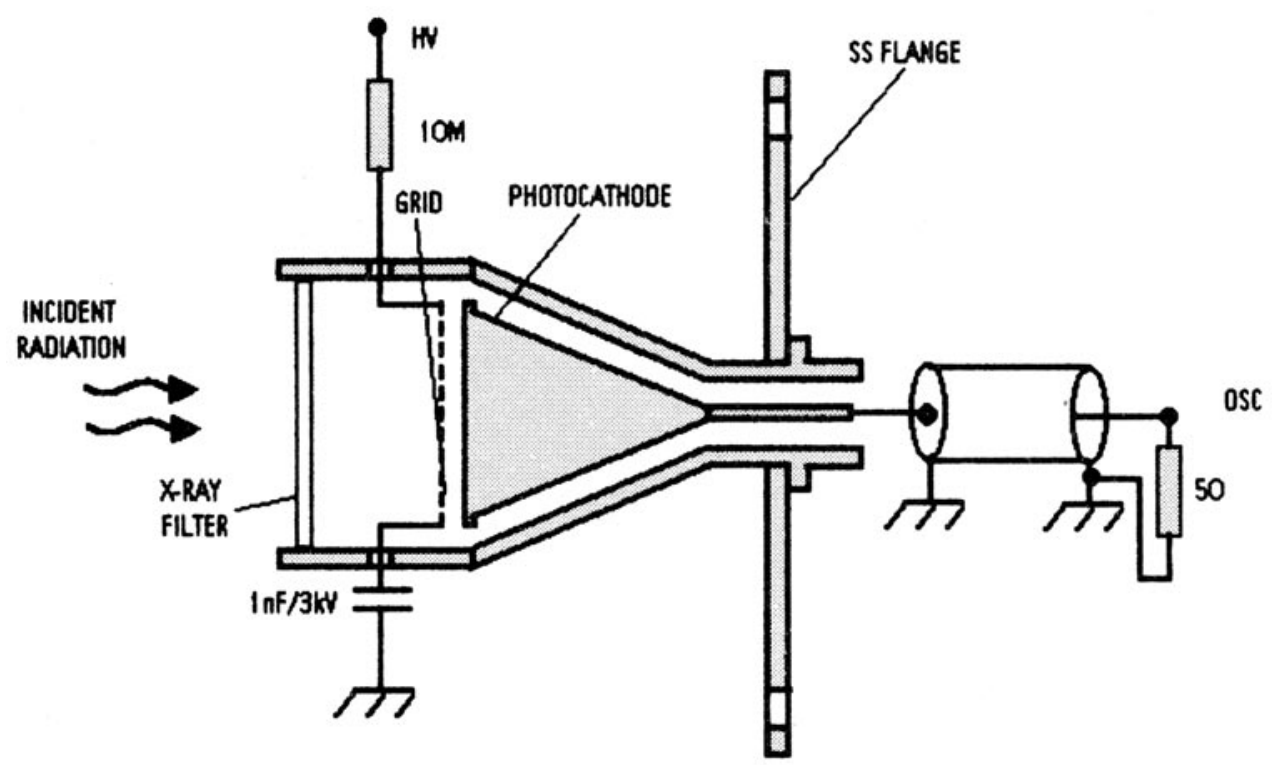

Figure 1. Schematic diagram of photodiode.

\subsection{Design of vacuum photodiode}

2.1 a Theoretical aspect: Before describing the design of biplanar vacuum photodiode, it is important to understand the physical processes taking place inside the photodiode, when ultraviolet or X-ray photons interact with the photocathode. Figure 1 shows the schematic diagram of a photodiode where interaction of photons with photocathode releases electrons without any time lag and whose energies are decided by the wavelength of the incident photons. Due to biasing voltage $\left(V_{b}\right)$ present between photocathode and anode, an electric field $E=V_{b} / d$ accelerates the electrons towards the anode, where $d$ is the separation between electrodes. In the case of uniform electric fields, electron velocity rises linearly with time $t$. The rate of change of charge induces current in the circuit proportional to the electron velocity. The current induced by a single electron can be given by

$$
I=e V \text {, }
$$

where $e$ is the electronic charge and $V$ is the velocity of electron in electric field, which can be expressed as

$$
V=\left(e V_{h} / m_{e}\right) \cdot T, \quad 0<T<T_{r},
$$

where $m_{e}$ is the mass of the electron. Equations (2) and (3) clearly indicate that current rises linearly with time till the electrons reach the anode after travelling distance $d$ in time $T_{r}$. The expression for $T_{r}$ can be written with the help of (3) as

$$
T_{r}=d\left(2 m_{e} / e\right)^{1 / 2} \cdot V_{b}^{-1 / 2}=2.7 \times 10^{-6} d / V_{b}^{1 / 2} .
$$

The maximum value for $l$ can be given by

$$
I_{\max }=\left(2 e^{3} / m_{e}\right) 1 / 2 \cdot V_{b}^{1 / 2} .
$$


Equations (4) and (5) indicate that rise time is inversely proportional to the square root of the applied voltage between the electrodes, whereas peak current is directly proportional to the square root of biasing voltage. This means that higher biasing voltage is important for both rise times as well as induced signal. Figure 1 shows the equivalent circuit for the vacuum photodiode in which induced current flow through the capacitor formed due to interelectrode separation and a load resistance $R_{L}$. The signal $V_{S}$ thus generated across load $R_{L}$ rises during the electron flight time to its maximum value $\left(V_{S O}\right)$ and then decays exponentially with a time constant $\tau=R_{L} C$ which can be expressed as

$$
V_{S}=V_{S O} \exp \left[-\left(T-T_{r}\right) / \tau\right], \quad T>T_{r} .
$$

This indicates that the fall time of the induced signal is limited by the discharge time constant of the capacitor formed due to inter-electrode separation. It has been reported (Beck 1976) that the space charge limitation in the photodiode does not affect the response of the photodiode. The combination of equations above is capable of describing the response of the photodiode for the given parameters and the incident photon flux at any wavelength.

$2.1 \mathrm{~b}$ Design of single channel-vacuum photodiode: Figure 1 shows the schematic diagram of a single-channel biplanar vacuum photodiode (Shukla et al 1997a). It consists of a combination of grid and photocathode (dia $\sim 20 \mathrm{~mm}$ ). The grid has $\approx 80 \%$ transmission and forms the high-voltage electrode of the diode. It is placed on a brass ring separated from the photocathode by a teflon annular ring of thickness $0.5-1 \mathrm{~mm}$. The grid is connected to high voltage through an isolating resistance of $10 M \Omega$. A $1 \mathrm{nF} / 3 \mathrm{KV}$ ceramic capacitor is connected between grid and ground to improve the low frequency response of the diode, while the high-frequency response of the diode is limited by the transit time of photoelectrons between the photocathode and grid as well as by the stray inductance present in the circuit. The photocathode as well as the grounded housing of the diode are of conical geometry ( $50 \Omega$ transmission line) as shown in figure 1 to match that of the $50 \Omega$ BNC connector from where the output signal is taken. The outer housing is made of brass whereas the photocathodes are made of aluminum or gold-plated copper. The surface finish of the photocathode should be such as to provide long-term stability in quantum efficiency because rough surfaces encourage oxidation. An arrangement is made to place an X-ray filter attached to an annular teflon ring in front of the grid in order to select the spectral range of the X-rays being detected. Output signals are recorded across $50 \Omega$ from the photocathode using a fast oscilloscope to avoid their reflection. However to improve the $S / N$ ratio of the diode one can increase the sensitivity of the detector either by increasing the area of the photocathode or by increasing the value of the termination resistance at the cost of the time response of the detector.

2.1c Design of multichannel vacuum photodiode: Figure 2 shows the schematic diagram of a multichannel vacuum photodiode (Rai et al 1995b) which consists of four vacuum photodiodes in one assembly. The only difference in this case is that the photocathode has a disc of $\approx 10 \mathrm{~mm}$ diameter and a pin coming out from the disc making $50 \Omega$ coaxial transmission line geometry with the common metal housing for all the four photodiodes. However, some mismatch remains between the photocathode pin and the BNC connector near the electrical joints. These photodiodes are operated in two modes, in one case voltage is applied on the grid and the signal is taken from the photocathode across the $50 \Omega$ termination, while in the other, voltage is applied on the photocathode and the grid is grounded, where output signal from the photocathode is taken through a capacitor to isolate 


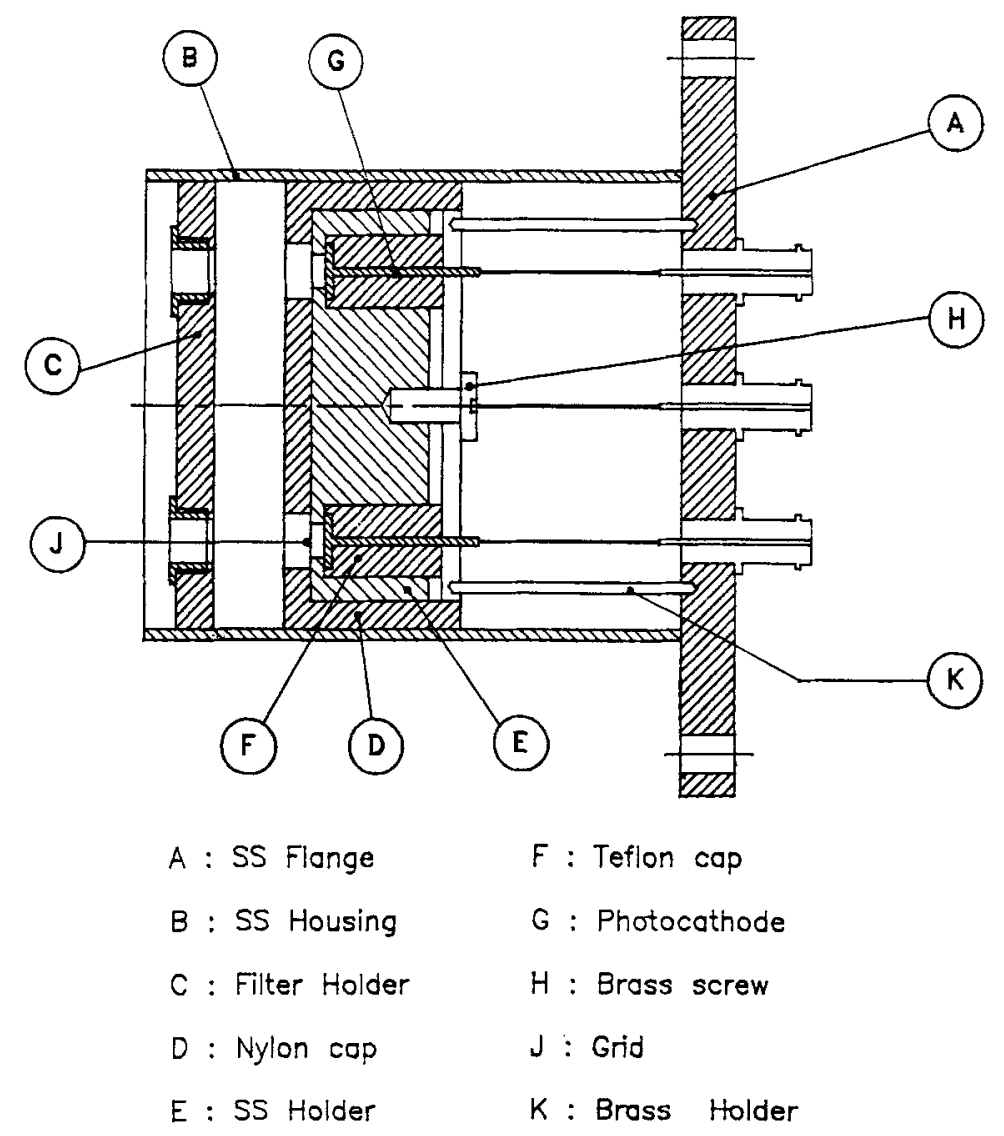

Figure 2. Schematic diagram of multichannel vacuum photodiode.

the DC voltage on the input of the oscilloscope. X-ray signals are recorded using multichannel photodiodes in different energy ranges (in a single shot) by using different types of $\mathrm{X}$-ray filters in front of the grids of the photodiodes. Both the systems are assembled within the flange of the vacuum chamber, while output signals are taken out through the vacuumtight feedthrough.

\subsection{Testing of vacuum photodiode}

These vacuum photodiodes are tested using an X-ray source obtained from the laserproduced plasma. For this a picosecond laser is focused on a copper target kept in a chamber evacuated at $\sim 10^{-5}$ torr in which vacuum photodiodes are also connected through the ports. Figure 3a shows the oscillogram of signals obtained from a single-channel vacuum photodiode when X-rays fall on the photocathode (Shukla et al 1997a). Picosecond laser signals recorded using a PIN-photodiode are also shown for comparison in the same oscillogram. Both signals have similar rise time, nearly $\approx 1 \mathrm{~ns}$, which is limited by the sampling rate of the oscilloscope ( $1 \mathrm{G}$ sample, LeCroy model 9350A). A fast oscilloscope is needed to record the subnanosecond phenomenon because the calculated response time of the photodiode is $T_{r}(10-90 \%)=0.8 d\left(2 m_{e} / e V_{b}\right)^{1 / 2} \cong 130 \mathrm{ps}$ for biasing voltage of $\approx 100$ volts and electrode separation of $0.5 \mathrm{~mm}$. It is necessary to have a fast oscilloscope if 
4-Jul-96

$15: 23: 17$

(a)
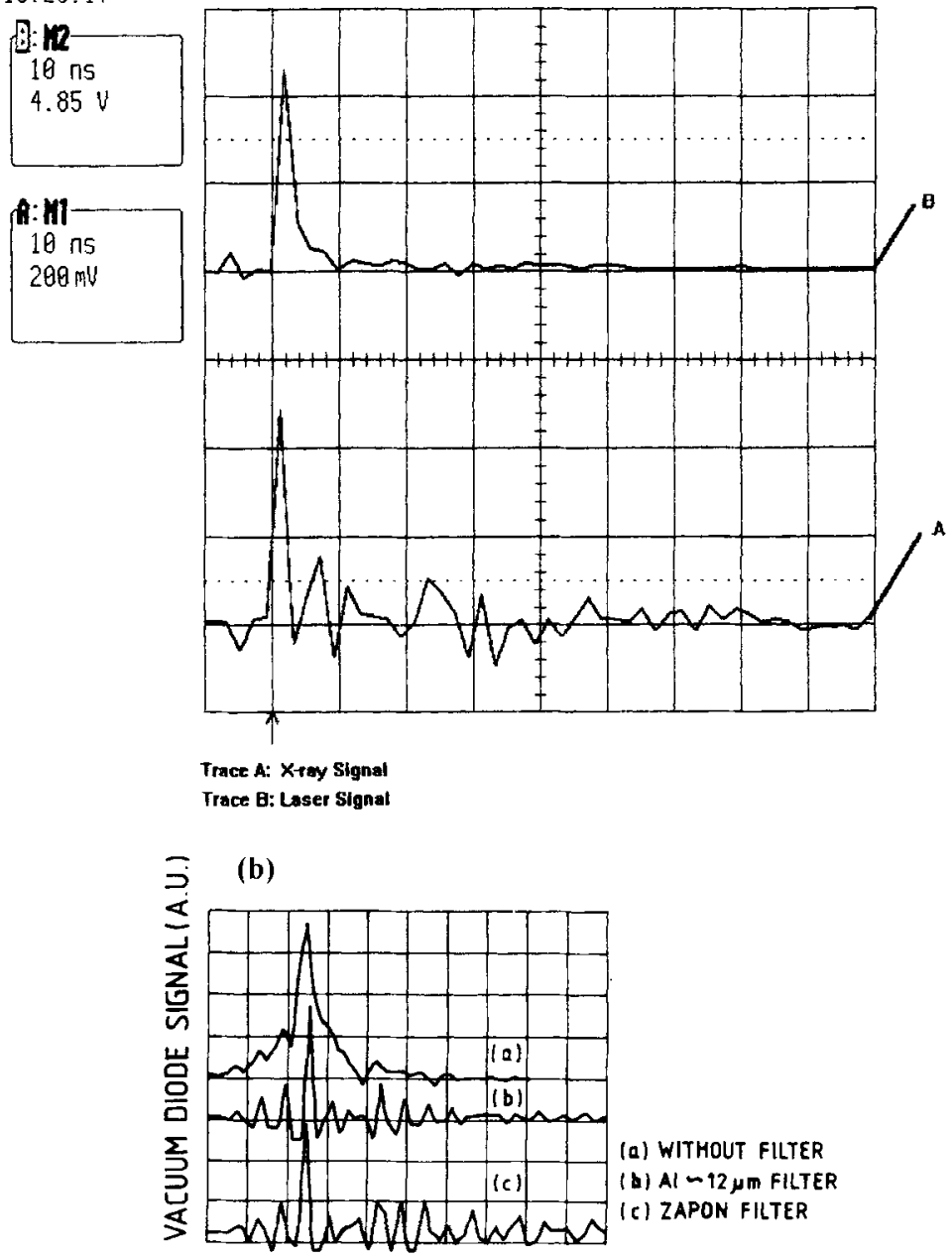

TIME ( 12.5 ns/DIV.)

Figure 3. Oscillogram of signals obtained from (a) single channel vacuum photodiode, and (b) multichannel vacuum photodiode.

the temporal response of X-ray emission is to be recorded in the picosecond regime using these types of photodiodes. Otherwise it provides time-independent integrated information. Figure $3 \mathrm{~b}$ shows the oscillogram of three photodiode signals from a multichannel photodiode assembly (Rai et al 1995b). Out of the three traces. the first one (a) is recorded using a photodiode without any filter, whereas the other two traces (b) and (c) are recorded in the presence of $\approx 4 \mu \mathrm{m}$ thick Zapon and $12 \mu \mathrm{m}$ thick aluminum filter respectively. In the former, the detector is sensitive to ultraviolet as well as $X$-rays, which remain for a longer time $>20 \mathrm{~ns}$, whereas in the latter case (presence of Zapon and aluminum filter) it is sensitive only to soft and hard X-rays, which show the time duration of the pulse as $\sim 3 \mathrm{~ns}$, which is again limited by the oscilloscope sampling rate (L\&T Gould Model 7074). The time durations of UV and X-ray emissions recorded using photodiodes are in agreement with the measurements made using an X-ray streak camera (Rai et al 1995a) where 
ultraviolet and $\mathrm{X}$-rays emissions last for $>20 \mathrm{~ns}$ (without filter) and soft $\mathrm{X}$-rays last for $1.5 \mathrm{~ns}$ (with Zapon filter) (Rai et al 1998a). During these experiments, photodiodes are biased to $\approx 700$ volts (optimum voltage) and signals are recorded across $50 \Omega$ termination. In figure $3 \mathrm{~b}$ one can observe the presence of two smaller peaks before the main peak for all the photodiode records. These pulses are due to the presence of two mode-locked prepulses before the main laser pulse, separated by $7 \mathrm{~ns}$ time duration. These multiple modelocked pulses are noticed when the acousto-optic modulator of the picosecond laser was not properly aligned. However, during the best alignment case also, one can see two smaller laser pulses before and after the main laser pulse separated by $7 \mathrm{~ns}$. The important observation in this case is that the main X-ray signal increases when two prepulses are present with significant amplitude, which can generate preformed plasma before the arrival of the main laser pulse. However comparatively fewer X-ray signals are observed when only one laser pulse with smaller side bands is present during the best alignment of the laser. This indicates that expanded plasma, preformed during prepulse, interacts with the main laser pulse and provides good conditions for laser light absorption in plasma corona as well as plasma heating and consequently an increase in X-ray emission is observed. These photodiodes have been used to study the X-ray emission from laser-produced plasma expanding across a uniform magnetic field (Pant et al 1998; Rai et al 1998a). In this experiment, photodiode signals are recorded across $1 M \Omega$, that is, in the integration mode due to two reasons. First, to increase the signals generated by $\mathrm{X}$-rays and, second, to avoid the fluctuations in photodiode signals due to sampling and reflection as a result of impedance mismatches.

\section{Picosecond time resolution, $X$-ray and optical streak camera}

The streak camera is one of the most versatile instruments (Schelev et al 1972; Campillo \& Shapiro 1983; Tsuchiya 1983; Stradling et al 1983; Kinoshita et al 1983; Rai et al 1995a) today for high-speed photometry in the field of physics, chemistry, biology and nonlinear optics. Particularly, hydrodynamic and radiative processes in laser-produced plasma and inertial confinement fusion experiments with rapid time variations demand detailed studies of time resolved X-ray and optical emission from laser-produced plasma. Conventional optical detectors such as biplanar photodiodes and PIN silicon photodiodes used along with the fastest available oscilloscopes provide time resolutions of $\approx 10^{-10} \mathrm{~s}$. Various other techniques generally used for investigating very fast optical events are the autocorrelation method, second harmonic generation, two-photon fluorescence and four-photon parametric mixing. However, some percentage of errors (inaccuracies) is always associated with these measurements. Amongst all the available methods, streak photography is considered to be the best for recording ultrafast optical phenomena with excellent time resolution $(<1 \mathrm{ps})$. It can directly provide temporal profiles of any optical event even in single-shot operations. In addition to the time duration, rise time as well as shape of the optical pulse can also be recorded along with intensity modulation (fine structure) if present during the optical event. However, measurements are limited by the time resolution of the streak camera. Streak cameras with nanosecond time resolution were built long ago, but temporal resolutions of picosecond or sub picosecond order have been achieved only recently. New streak cameras with femtosecond time resolutions have also been reported (Kinoshita et al 1987; Guide \& Novokhatsky 1995). Highly sensitive streak cameras with picosecond time resolutions are now commercially available and are capable of recording any phenomena in the spectral 
range extending from near-infrared to ultraviolet and soft $\mathrm{X}$-ray $(100 \mathrm{eV}-10 \mathrm{keV})$ regions with the help of suitable photocathode attachments. In fact, the streak camera can functionally be referred to as a $>300 \mathrm{GHz}$ bandwidth optical oscilloscope. However, the streak camera possesses many more special features, which help it score over an ordinary oscilloscope, for example, it can provide three-dimensional information such as spatially time-resolved profiles as well as time-resolved spectroscopic intensity profiles (Campillo \& Shapiro 1983; Tsuchiya 1983). Either a photographic system or a digital image memory is used to store and analyse three-dimensional streak images, whereas the latter provides nearly real-time measurements of the events.

\subsection{Streak tubes}

We have used the optical and X-ray streak and image intensifier tubes manufactured by General Physics Institute, Moscow for making our streak cameras. The optical streak camera has a S1 type photocathode (Ag-O-Cs) deposited on a flat glass plate of $5 \mathrm{~mm}$ diameter and is sensitive in the spectral range $450-1150 \mathrm{~nm}$ with a peak response at $800 \mathrm{~nm}$. The input end of the X-ray streak tube (also obtained from the Institute of General Physics, Moscow) is attached to a metal flange, which has a clearance for the photocathode at the centre and a number of small holes around it to evacuate the tube. Generally, an X-ray streak tube has a demountable photocathode and is evacuated directly during experiments. The rest of the parts of the tube are similar to those of the optical streak tube. The X-ray sensitive photocathode consists of a $300 \AA$ thick gold film deposited on a $1000 \AA$ thick parylene or nitrocellulose film fixed to the cathode disc which has a $100 \mu \mathrm{m} \times 9 \mathrm{~mm}$ slit. This photocathode is sensitive to soft $\mathrm{X}$-rays ranging from $100 \mathrm{eV}$ to $10 \mathrm{keV}$ as well as to UV radiation. Instead of gold, a CsI photocathode provides better quantum efficiency but is hygroscopic in nature, which makes this type of photocathode very sensitive from the point of view of storage. The slit image produced by the incident soft X-rays is focused onto the phosphor screen by the electron lens made by the combination of accelerating mesh, focusing electrode and anode. An accelerating grid placed at a distance of $1 \mathrm{~mm}$ from the photocathode has $60 \%$ transmission. The acceleration grid is useful in reducing the time resolution limit of the streak camera to $\leq 1 \mathrm{ps}$. The focusing electrode focuses the photoelectrons on to the phosphor screen and decides the spatial resolution of the tube. The photocathode, accelerating grid and focusing electrodes require a biasing voltage of -15 , -13 and -12 to $-13 \mathrm{kV}$ respectively. It has an anode which acts as an aperture in the tube and remains at the ground potential. There are two pairs of deflector plates out of which one pair is used to sweep the photo electrons on to the screen whereas the other pair is used to fly the photo electrons back to their initial position without streaking back on to the screen to the position from where sweeping started. The phosphor screen of the camera is made up of aluminized P11 material, which has a peak response at $460 \mathrm{~nm}$ (blue region) and is coupled to the output face of an optical fibre plate. The optical streak tube is a sealed-off type with a glass window on the photocathode side. An image intensifier tube is coupled to the streak tube as shown in figure 4. The image intensifier tube consists of a photocathode, a phosphor screen and a microchannel plate (MCP) to multiply the photoelectrons. This system is used to enhance the intensity of the image obtained at the phosphor screen of the streak tube. It has a maximum gain of $\sim 10^{4}$ divided into 5 steps. Finally, the image on the screen of the intensifier is recorded by using contact photography or a CCD camera. 


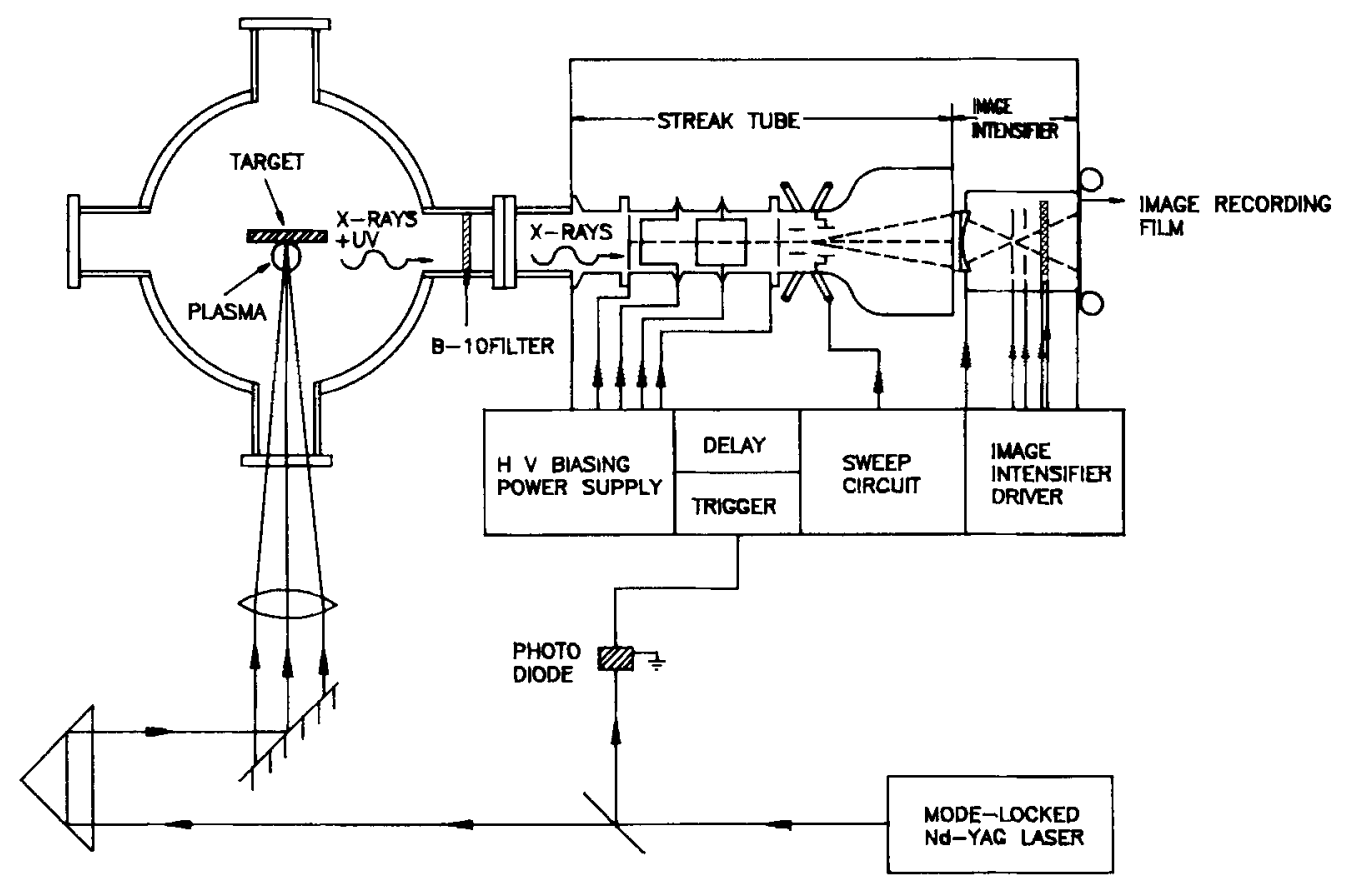

Figure 4. Experimental set-up for testing the X-ray streak camera.

\subsection{Electronics}

The peripheral electronics of a streak camera contain various kinds of fast and high as well as low voltage pulser circuits. The main high voltage pulse requirements for the operation of streak camera are (i) a high voltage $(\sim 1 \mathrm{kV})$ linear ramp of both polarities for the full deflection of the photoelectrons across the $25 \mathrm{~mm}$ image intensifier screen (this is considered to be the heart of the streak camera), (ii) a short rectangular pulse of variable time duration $(100 \mathrm{~ns}-2 \mu \mathrm{s})$ and amplitude $(\max 2 \mathrm{kV})$ to gate the accelerating grid as well as to increase the time resolution of the camera (this is the optional system), (iii) a trigger pulse generator, which triggers the sweep circuit; however, this generator is externally triggered by a fast photodiode signal, and (iv) the last but important components of the streak camera are many high voltage $d c$ power supply sources used to bias various electrodes of streak and intensifier tubes such as photocathode, accelerating grid and focus electrode as well as to drive the sweep and gate pulse circuits.

3.2a Sweep pulse circuits: A picosecond time resolution is obtained in a streak camera by streaking the photoelectrons with a streak velocity $\sim 10^{10} \mathrm{~cm} / \mathrm{s}$. This indicates the need for a sweep voltage having a rise time $\leq 1 \mathrm{~ns}$. The rise time obtained using an RLC integrated circuit is normally slower than this. In order to prevent image distortion and improve camera dynamics as well as spatial resolution, two symmetrical well-balanced sweep pulses are required, which in fact make the sweep pulse faster as compared to the single sweep in the same rise time. The nonlinearity in the sweep pulse and, as a result, the sweep speed improve on increasing the amplitude of the sweep pulse beyond the required value (overpulsing). Many circuits have been reported for generating a sweep pulse for the 


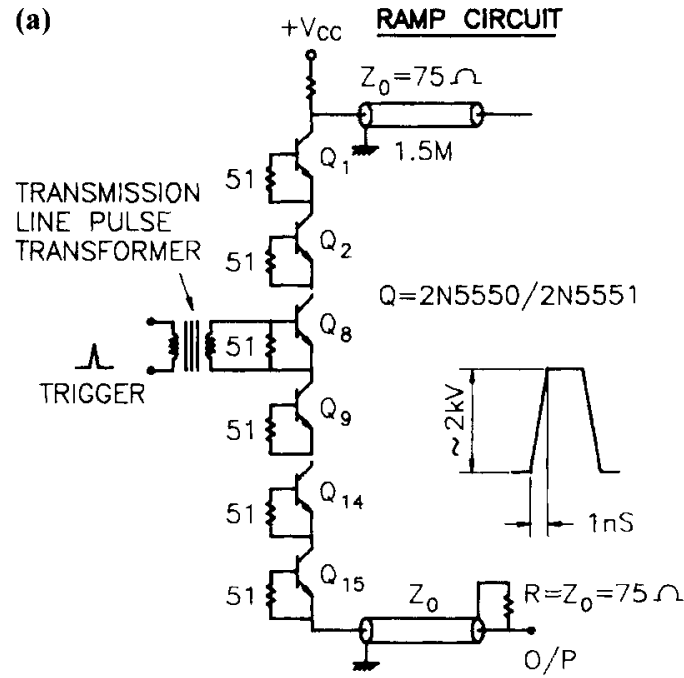

(b)
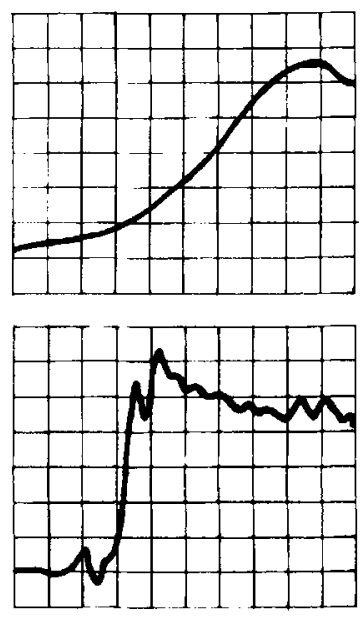

(II)

Figure 5. (a) Diagram of sweep pulse circuit using series stack of transistors; (b) oscillogram of sweep pulse from the series stack circuit. Scale - Horizontal: (I) $200 \mathrm{ps} /$ div., (II) $2 \mathrm{~ns} / \mathrm{div}$; vertical: (for both) $400 \mathrm{~V} /$ div.

streak camera. All these utilize various kinds of fast switching elements such as Krytron (Bradley et al 1972); power beam microwave triode, laser-triggered spark gap (Alcock et al 1970; Schelev et al 1972) as well as avalanche transistors (Cunin et al 1980; Baker 1991). The transistorized circuits available today use transistors in a series stack mode or in a Marx bank configuration for switching the circuit. A pulsar circuit was developed (Rai \& Shukla 1994) using a stack of 15 avalanche transistors $2 \mathrm{~N} 5551$ connected in series as a single switching element which is shown in figure 5a. A transmission line of $75(50) \Omega$ (impedance and $\sim 1.5 \mathrm{~m}$ in length was charged to $4.5 \mathrm{kV}$ and discharged through a $75(50) \Omega$ load. We obtain a square step pulse of $\sim 2 \mathrm{kV}$ across $75(50) \Omega$ resistor, instead of $\sim 2.25 \mathrm{kV}$ as a result of a small voltage drop across the transistors due to its finite resistance during the breakdown. This circuit provides a square pulse of rise time $800 \mathrm{ps}(10 \%-90 \%)$ as shown in figure $5 \mathrm{~b}$ when recorded on Tektronix oscilloscope (model 7104) having a vertical plug in model 7A 29 which had a combined bandwidth of $1 \mathrm{GHz}$. To achieve a rise time better than $1 \mathrm{~ns}$, along with a series combination of-transistors, reduction in excess leads and proper soldering are needed to reduce the extra inductance in the circuit. A carefully designed printed circuit board was prepared for this purpose. This circuit is triggered through a wide band pulse transformer, which consists of a small toroidal ferrite core with five turns of $50 \Omega$ coaxial cable, where the cable shield acts as a primary and the central conductor as a secondary. Triggering of any transistor in the stack switches the circuit, but better stability is obtained when a middle transistor of the stack is triggered. The triggering pulse is sharpened using a $20 \mathrm{PF}$ capacitor and a $50 \Omega$ resistance at the output of the transmission line pulse transformer in a differentiation mode. A small pulse width of the trigger pulse effectively protects the base emitter junction of the transistor being triggered and as a result enhances the lifetime of the circuit. Many earlier reported circuits (Cunin et al 1980; Baker 1991) use ballast resistance between emitter and collector of each transistor to avoid self-triggering in the circuits. However we did not observe any self-triggering in our circuit even without the ballast resistances. Nevertheless, a properly designed ballast 
(a)

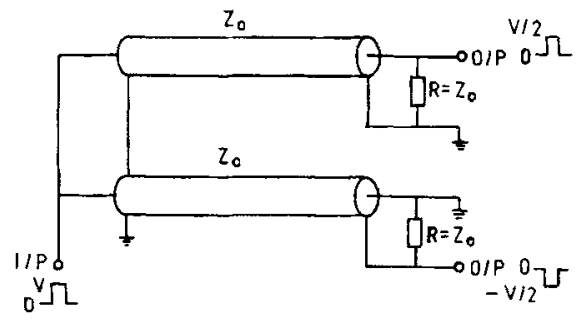

(b)

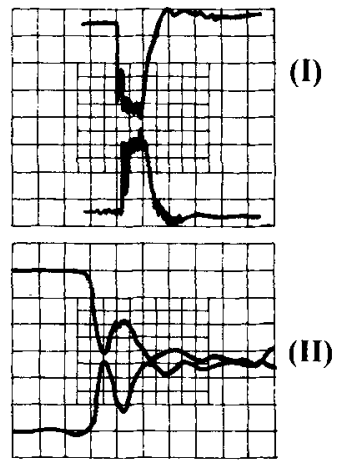

Figure 6. (a) Network to find two pulses of opposing polarities from one pulse; (b) oscillogram of the two pulses. Scale - Horizontal: (I) $10 \mathrm{~ns} / \mathrm{small}$ div., (II) $1 \mathrm{~ns} / \mathrm{small}$ div; vertical: (for both) $160 \mathrm{~V} /$ small div.

circuit may decrease the high jitter $(<500 \mathrm{ps})$ observed in this case to $<100 \mathrm{ps}$ and may keep the string from self triggering over a wide temperature range.

A simple technique (Rai \& Shukla 1994) is used for generating two symmetrical opposite polarity pulses from a single pulse of either polarity. This arrangement is shown in figure $6 \mathrm{a}$, and has two similar length $(2 \mathrm{~m}), 75(50) \Omega$ impedance cables connected in parallel at the output of the pulser circuit and terminated with $75(50) \Omega$ resistance. Towards the termination end, the first cable is grounded at the shield and the output is taken from the central pin, which provides a similar polarity pulse, like input pulse, but around one half in amplitude. The second cable has its central pin grounded at the termination end and the output is taken from the shield of the cable (figure 6a) which provides an opposite polarity pulse in comparison to the input pulse and similar in amplitude as is obtained from the first cable. Care must be taken to isolate the shield of the later cable with the normal ground at the termination end, otherwise the rising edge of the pulse becomes distorted. Figure $6 \mathrm{~b}$ shows the two symmetrical opposite polarity pulses of rise time better than $1 \mathrm{~ns}$. In the case of application of these pulses in streak camera only the rise time, amplitude and a small delay are important parameters of the pulser circuit. These opposite polarity pulses are used to operate the streak camera. However, it is noticed that the rise time of the pulse is modified to $\sim 2.5 \mathrm{~ns}$ due to capacitive loading from the deflection plate. The superimposed fluctuations observed on the pulses in figures $5 \mathrm{~b}$ and $6 \mathrm{~b}$ appear to be due to the unwanted signal travelling on the outside of the outer conductor. These fluctuations can be removed or minimized by placing the circuit in a properly shielded box or by wrapping the coaxial cable on a large ferrite core. The signals on the outside of the outer conductor will travel at a slower velocity due to the high $\mu$ of the ferrite. For the slow streak velocity, an RLC integrator is used at the output of the circuit. With this circuit, streak camera provides a streak rate of $15 \mathrm{~mm} / 1 \mathrm{~ns}$.

Another circuit shown in figure 7 works on the principle of Marx bank switching scheme (Rai et al 1994d; Shukla et al 1997b) in which capacitors $(470 \mathrm{pF} / 2 \mathrm{kV}$ ) are charged in parallel and then discharged in series by triggering one of the transistors $2 \mathrm{~N} 5551$ in stack number 4. A pulse generator applies the trigger pulse through pulse transformer. The lifetime of the sweep circuit based on Marx bank configuration increases due to low biasing voltage (1100 volts) as compared to the circuit based on series stack configuration of avalanche transistors as discussed above. Due to the large number of stages in Marx 
(a) $+V c c$

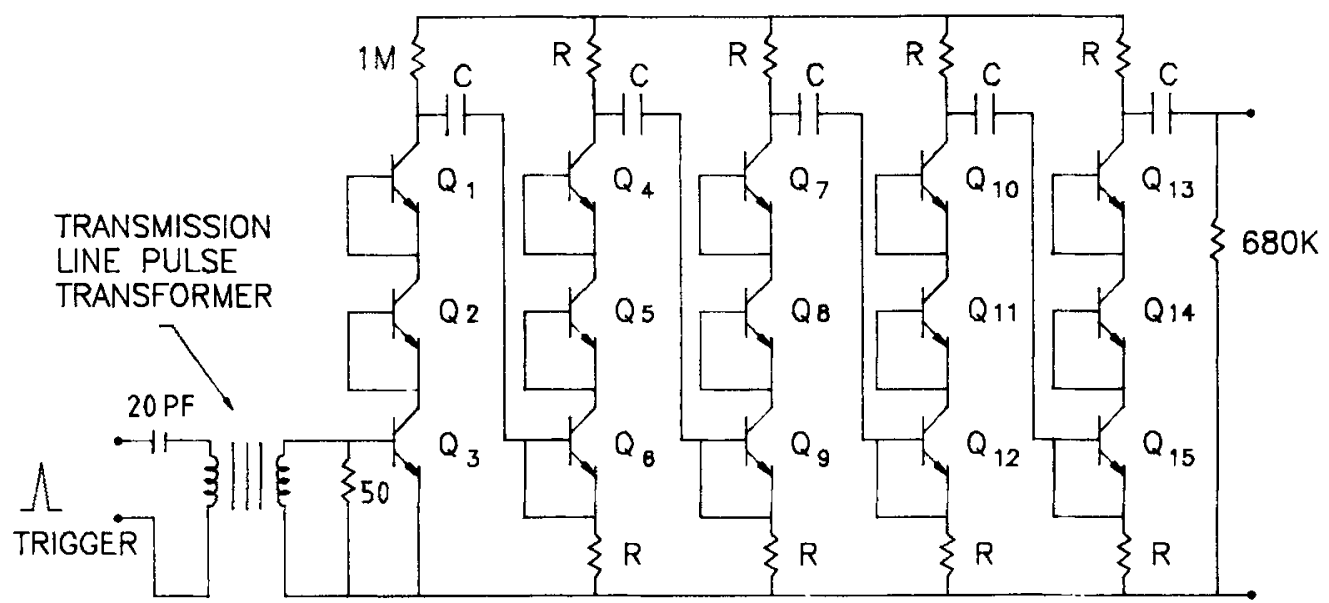

(b)
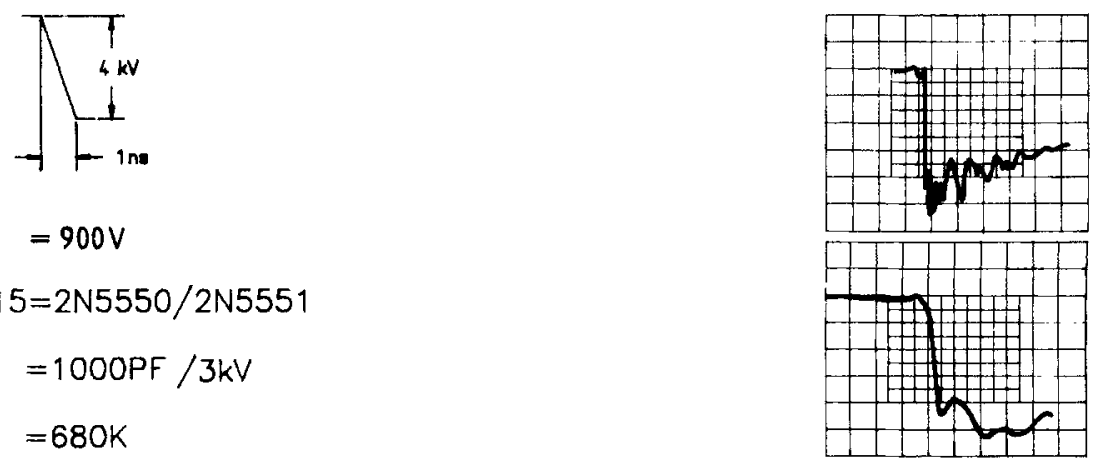

$V_{c c} \quad=900 \mathrm{~V}$

$$
\begin{aligned}
\text { Q1-Q15 } & =2 \mathrm{~N} 5550 / 2 \mathrm{~N} 5551 \\
\mathrm{C} \quad & =1000 \mathrm{PF} / 3 \mathrm{kV} \\
\mathrm{R} & =680 \mathrm{~K}
\end{aligned}
$$

Figure 7. (a) Sweep circuit using Marx bank; (b) oscillogram of sweep voltage. Scale - Horizontal: (I) $10 \mathrm{~ns} / \mathrm{small}$ div., (II) $1 \mathrm{~ns} / \mathrm{small} \mathrm{div}$; vertical: (for both) $400 \mathrm{~V} / \mathrm{small}$ div.

bank circuit (modified circuit has 7 stacks with 3 transistors in one stack), sweep voltage generated is large $(\sim+2.5 \mathrm{kV})$ with rise time $\leq 2.5 \mathrm{~ns}$ whereas in series stack switching, charging voltage needed is $4.5 \mathrm{kV}$ out of which half the voltage drops due to transmission line properties and is further reduced to only $+1 \mathrm{kV}$. A portion of the ramp voltages $( \pm 500 \mathrm{kV})$, selected by applying positioning DC voltage of \pm 1300 volts, are applied to the pair of deflection plates. This sweep voltage is further sharpened using $L C R$ pulse-shaping passive components between the generator and deflection plates with the help of a 6-way high voltage rotary switch specially designed for this purpose. The $L C R(L=3.6 \mathrm{nH}, C$ is deflection plate capacitance, $R=470 \mathrm{ohms}$ ) combination provides full-screen deflection in $\approx 700 \mathrm{ps}$ as compared to $2.5 \mathrm{~ns}$ in the series stack circuit. The sweep voltage is also integrated using different values of $L, C$ and $R$ to provide full-screen deflection in 1.2, 3, 5, 8 and $20 \mathrm{~ns}$ for slower streak velocities. Thus, as compared to the series stack circuit, this circuit also generates fast sweep pulses but with better stability which helps in fast sweeping of photoelectrons across the phosphor screen with better temporal resolution. Further, the circuits shown in figure 7 are directly mounted on the deflection plates. This 

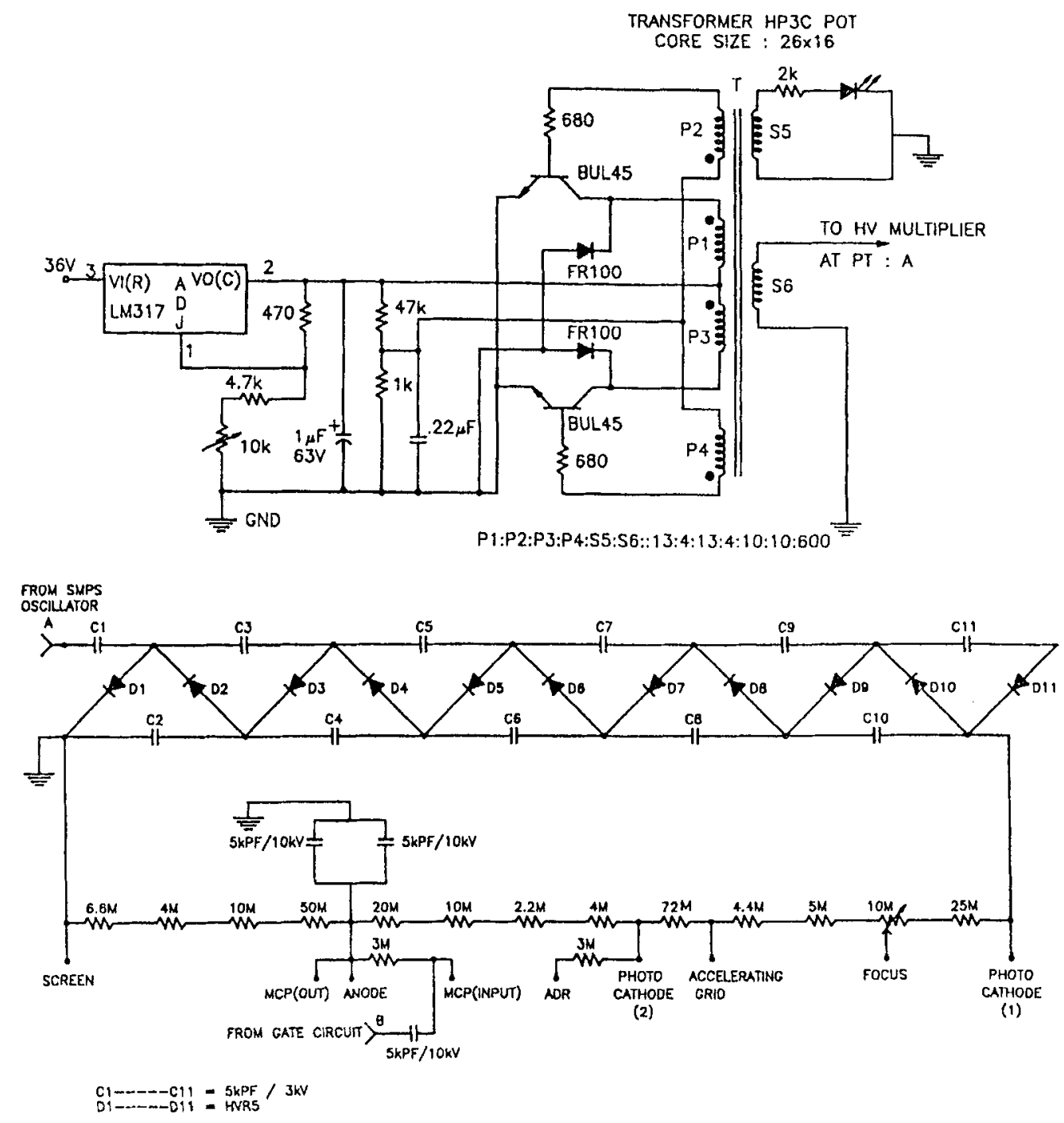

Figure 8. Switch mode power supplies to bias the streak and intensifier tubes.

reduces the trigger delay and also the inductive and capacitive loading of the circuit and provides a streak rate of $35 \mathrm{~mm} / \mathrm{ns}$.

These high voltage pulser circuits are useful for many other applications also such as driving the pockel cell for mode locking and $Q$ switching of solid state lasers.

3.2b High voltage dc power supply: Several high voltage DC power supplies are needed to drive the picosecond streak camera. The photocathode, accelerating grid and focusing electrodes of X-ray streak camera require biasing voltages of $-15,-12$ and $-13 \mathrm{kV}$ respectively with respect to the anode and the phosphor screen. A 4.5 or $2 \mathrm{kV}$ power supply drives the sweep circuit and the gate circuit whereas a variable \pm 1300 volts supply controls the position of streak on the screen. Various switch mode power supplies (SMPS) having compact sizes have been made for the above applications. Figure 8 shows the circuit 
diagram of the high voltage switch mode power supply (SMPS) oscillator. Switching transistors BUL45 work on the principle of gain-limited switching. The transformer of the SMPS oscillator is wound on a ferrite pot core (HP3C material) of size $26 \times 16$ which consists of 13 and 4 bifillar turns of SWG30 copper enamelled wire connected at the collector and base of the transistors respectively. The secondary of the transformer consists of 600 turns (SWG38 copper wire) in 10 layers where each layer consists of 60 turns insulated by teflon tape. The final voltage at the output of the transformer is multiplied to $-15 \mathrm{kV}$ whereas the required biasing voltages for biasing the intensifier tube are obtained by using resistive dividers. Detailed design have been reported elsewhere (Shukla et al 1996).

\subsection{High speed high voltage probes}

Various applications of high speed high voltage pulsers such as drivers for streak cameras as discussed above, pockel cells and optical modulators require reliable high voltage probe and attenuators. These fast probes act as developmental diagnostics or as continuous monitoring systems to obtain the pulse shapes from pulser circuits. Many designs of capacitive probes providing time response of a few nanoseconds (frequency response from a few megahertz to $300 \mathrm{MHz}$ ) with an attenuation factor of about $\sim 10^{8}$ have been reported together with the analysis of their performances and sources of errors. A new and simple capacitive divider was fabricated having attenuation of about $\sim 1600$ and frequency response extended to $>350 \mathrm{MHz}$. In addition to this, coaxial attenuators are widely used as dividers to reduce the input signal to a recording device such as a digitizer or an oscilloscope and are advantageous owing to their large bandwidth, low power dissipation, compact size and minimum reflection. In view of these requirements, a few attenuators also have been developed (Rai et al 1994b) with such characterstics like the Tektronix model

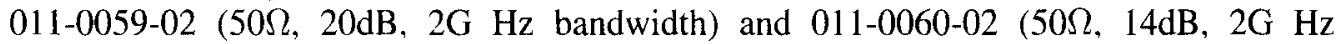
bandwidth).

3.3a Design of capacitive divider: Figure 9a shows the simple circuit of a capacitive divider in which capacitor $C_{1}$ and $C_{2}$ act as the first stage of the device and the resistances $R_{i}$ and $Z_{0}$ form the second stage of the divider when signals are recorded across $Z_{0}=50 \Omega$ termination. $C_{1}$ and $R_{i}$ are variable in the divider and decide the attenuation of the divider. The capacitor $C_{2}$ provides internal inductance $L_{i}$ and stray inductance $L_{2}$ in the circuit. If $V_{1}$ is to be measured and $V_{2}$ is the voltage observed across $R=\left(R_{i}+Z_{0}\right)$, then the circuit acts as a simple capacitive divider. A simple analysis shows three cases for its operation as below.

Case 1 - When $R\left(C_{1}+C_{2}\right) \ll t_{r}$,

$$
\mathrm{d} V_{1} / \mathrm{d} t=V_{2} / R C_{1} \text {. }
$$

$T_{r}$ is the characteristic time of any change in $V_{1}$. Here the measured signal is proportional to the change in voltage with respect to time.

Case 2 - When $R\left(C_{1}+C_{2}\right) \gg t_{r}$,

$$
V_{1}=\left(C_{1}+C_{2}\right) / C_{1} \cdot V_{2} \text {. }
$$

In this case it acts as a capacitive divider. 


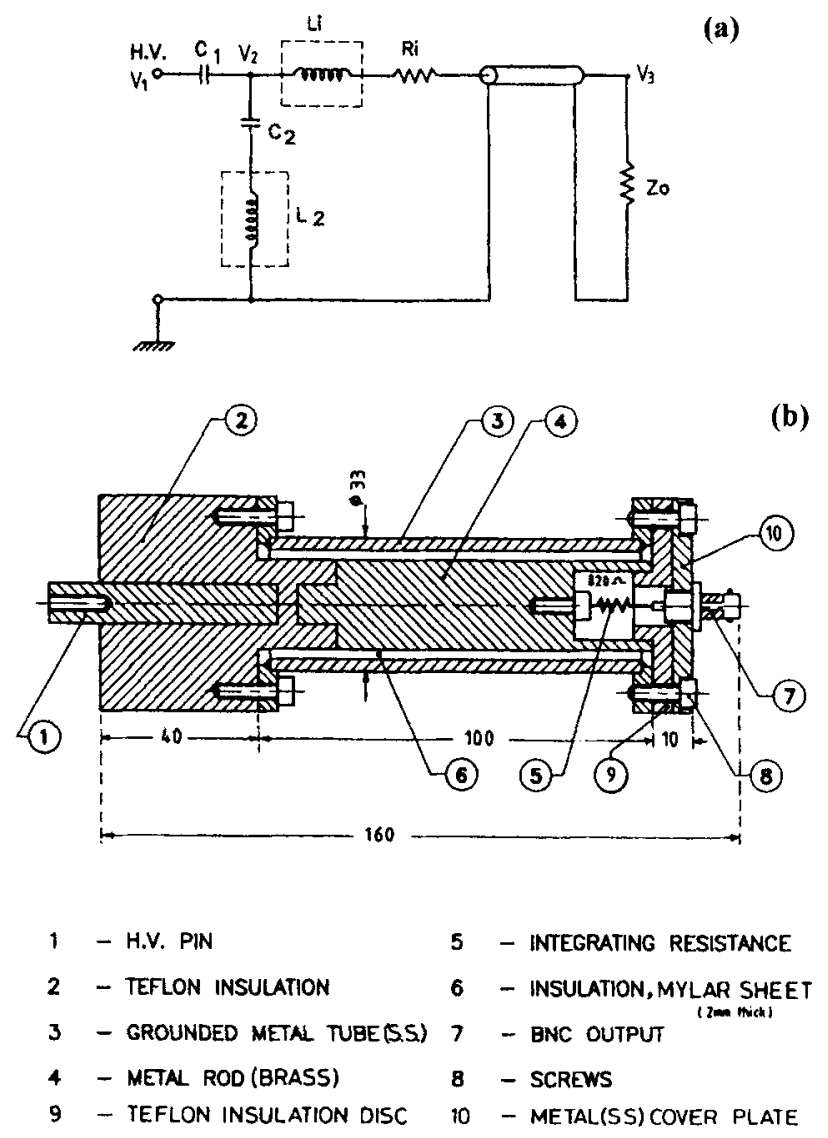

Figure 9. (a) Circuit, and (b) cross-sectional diagrams of capacitive divider. $\mathrm{R} 1=\mathrm{R} 2=38 \mathrm{ohms}, \mathrm{R} 3=12 \mathrm{ohms}$.

Case 3 - When the output voltage is measured across $Z_{0}$ as $V_{3}$, resistances $R_{i}$ and $Z_{0}$ act as the second stage of the divider and the total attenuation factor $(A)$ is approximated by

$$
A=V_{1} / V_{3}=\left(C_{1}+C_{2}\right) / C_{1} \cdot\left(R_{i}+Z_{0}\right) / Z_{0} .
$$

Figure $9 \mathrm{~b}$ shows a longitudinal section of the capacitive divider. In this $C_{2}(43 \mathrm{pF})$ is made from a brass rod (OD $22 \mathrm{~mm}$ ) inserted in an axially symmetric tube (ID $27 \mathrm{~mm}$ ) which is separated using thin mylar sheets. $C_{2}$ acts as a coaxial transmission line. The length of $C_{2}$ is $10 \mathrm{~cm}=\lambda / 4$ for frequencies $>350 \mathrm{M} \mathrm{Hz} . C_{1}$ is formed between the bottom of the pick-up pin $(\sim 10 \mathrm{~mm}$ diameter rod) inserted into a teflon cup and the flat portion of the inner electrode, which is a portion of $C_{2}$ with a $5 \mathrm{~mm}$ thick teflon layer in between acting as an insulator (figure 9b). Detailed information about this probe has been reported earlier (Rai et al 1994b).

$3.3 b$ Design of attenuators: $\quad T$ - and $\pi$-type attenuators kept between driving source and load provide an attenuation factor $(A)$, which is inversely proportional to the insertion loss. An expression for insertion loss $L_{i}$ in case of the $T$-type attenuator can be written as

$$
L_{i}=I_{2} / I=R_{3}\left(R_{3}+R_{L}\right) /\left[\left(R_{S}+R_{1}+R_{3}\right)\left(R_{2}+R_{3}+R_{L}\right)-R_{3}^{3}\right],
$$



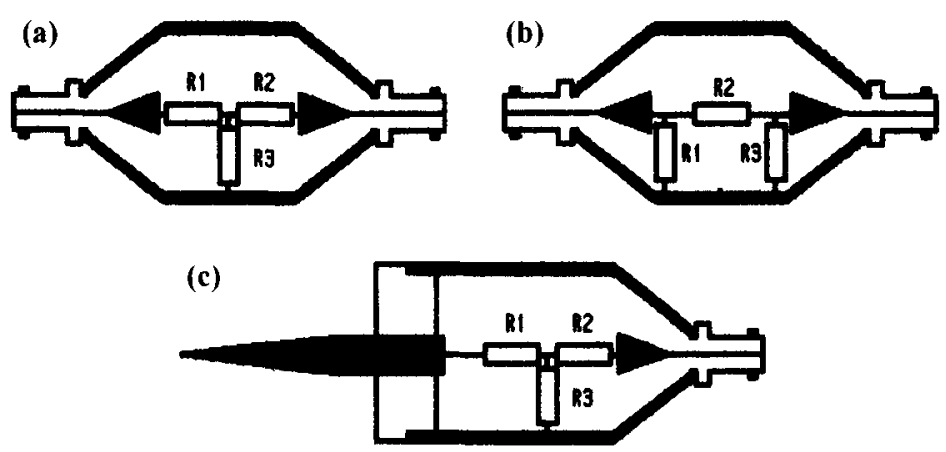

Figure 10. (a) T-type attenuator; (b) $\pi$-type attenuator $-\mathrm{R} 1=\mathrm{R} 3=50 \mathrm{ohms}$, $\mathrm{R} 2=2.5 \mathrm{k} \Omega$; (c) high input impedance resistive probe $-\mathrm{R} 1=\mathrm{R} 2=1 \mathrm{k} \Omega, \mathrm{R} 3=50 \mathrm{ohms}$.

where $I$ is the current in the circuit, when only source resistance $R_{S}$ and load resistance $R_{L}$ are present. $I_{2}$ is the current in load when a $T$-attenuator is inserted between load and source (figure 10a). In this case, insertion loss can be calculated from (10) after making $R_{1}=R_{2}=R$ and $R_{S}=R_{L}=R_{0}$. The values of $R$ and $R_{3}$ for required insertion loss are calculated as

$$
R=R_{0}\left(1-L_{i}\right) /\left(1+L_{i}\right), \quad R_{3}=2 R_{0} L_{i} /\left(1-L_{i}^{2}\right) .
$$

A balanced $\pi$-type attenuator (figure 10b) has $R_{1}=R_{3}=R$ and $R_{s}=R_{L}=R_{0}$ in (10).

The values of resistance for the $\pi$-type attenuator are calculated in terms of insertion loss as

$$
R=R_{0} \cdot\left(1+L_{i}\right) /\left(1-L_{i}\right), \quad R_{2}=R_{0} / 2 \cdot\left(1-L_{i}\right)^{2} / L_{i}
$$

In principle, these attenuators have frequency independent responses. For better performance of attenuators, however, resistances should be non-inductive and the whole circuit should be assembled in $50 \Omega$ coaxial geometry as shown in figure $10 \mathrm{~b}$.

A high input impedance resistive probe (figure $10 \mathrm{c}$ ) has also been fabricated, which has resistance $R_{1}=R_{2}=1 \mathrm{k} \Omega$ and $R_{3}=50 \Omega$ in a $T$-type configuration. The input connector in this probe is a high voltage pin. This probe provides an attenuation factor of about 400 . All the above mentioned probes provide sub-nanosecond responses and are used successfully in the development of sweep circuits of streak cameras. Oscillograms reported in figure $5 \mathrm{~b}$ and $6 \mathrm{~b}$ have been measured using only these probes.

\subsection{Temporal resolution of streak camera}

Temporal resolution of the streak camera is mainly dependent on three factors (Tsuchiya 1983).

(i) The transit time spread which is caused by the initial velocity distribution of the photoelectrons emitted simultaneously from the photocathode. This occurs in the low velocity domain between the photocathode and the mesh electrode and is given by

$$
\Delta t_{1}=2.3 \times 10^{-6}(\Delta \varepsilon)^{1 / 2} / E,
$$

where $\Delta \varepsilon$ is FWHM in energy distribution of the emitted photoelectrons which is $\sim 0.4 \mathrm{eV}$ when a photon of $904 \mathrm{~nm}$ falls on the $S_{1}$ photocathode. $E$ is the accelerating electric field near the photocathode which is $\sim 10^{6} \mathrm{~V} / \mathrm{m}$ in our case, and can provide a time spread $\Delta t_{1} \sim 1.5 \mathrm{ps}$. However, a small time spread will exist between mesh and the phosphor screen, which is very small compared to this time. 
(b) X-Axis : 118ps/100pixel

(a)

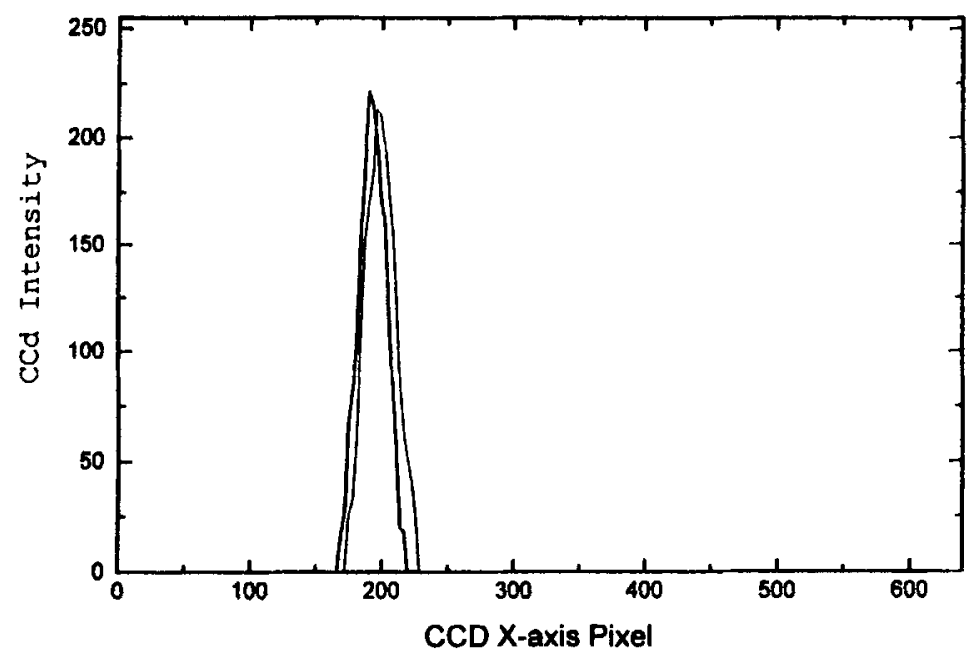

Figure 11. (a) Temporal evolution of Nd: YAG laser beam recorded using optical streak camera. Here the upper spot is due to delay in half the portion of the laser beam by using a $1 \mathrm{~mm}$ thick glass plate. (b) Intensity profile of the streaked image of the direct and delayed laser beam.

(ii) The technical or streak limited time resolution, given by

$$
\Delta t_{2}=1 / v \delta
$$

where $v \sim 3.5 \times 10^{10} \mathrm{~mm} / \mathrm{s}$ is the measured streak velocity and $\delta \sim 10 \mathrm{lp} / \mathrm{mm}$ is the measured spatial resolution of the streak camera. This provides $\Delta t_{2} \sim 2.86 \mathrm{ps}$.

(iii) The time spread $\Delta t_{3}$, which occurs due to the effect of deflection electric field on the photoelectron beam. It depends on the beam diameter, length of the deflecting plate, the deflection electric field and on the axial (directional) velocity of the electrons. This time spread is typically $\sim 1 \mathrm{ps}$. The actual time resolution of streak camera $\Delta t$ is given by

$$
\Delta t=\left(\Delta t_{1}^{2}+\Delta t_{2}^{2}+\Delta t_{3}^{2}\right)^{1 / 2},
$$

which is $\sim 3.38 \mathrm{ps}$. This is the estimated time resolution of the streak camera obtained using (15), which is in close agreement with the measured value of $\leq 5 \mathrm{ps}$ (figures $11 \mathrm{a}$ and $b$ ). The experimental limit of the time resolution is measured by keeping a glass plate of $1 \mathrm{~mm}$ thickness in half of the beam to optically delay the half portion of the image by $\sim 5$ ps (figure 11a). The image thus obtained clearly resolves the direct as well as the delayed pulse. The temporal resolution of an X-ray streak camera is greater compared to the optical streak camera even if the electronics are similar. This is due to the large value of $\Delta \varepsilon \approx 4 \mathrm{eV}$ for gold or CsI photocathode as is discussed in (i).

\subsection{Quantitative measurement using $X$-ray streak camera}

As discussed earlier the streak camera produces two-dimensional images. One dimension records the temporal history whereas the other dimension records the spatial variation of 
X-ray signals across the entrance slit. Quantitative information can be obtained from the streak measurement by correcting the signals recorded from the X-ray flux incident on the slit. If $F_{x}$ is the incident $\mathrm{X}$-ray flux, it is related to the X-ray streak camera signal $S$ by the expression

$$
S=\left[(d \xi \epsilon \gamma) /\left(m_{x} m_{t} v_{s}\right)\right] \cdot \eta F_{x},
$$

where $d$ is the entrance slit width, $V_{s}$ is the sweep speed of the camera at the output image and $\eta$ is the photocathode conversion efficiency. The image converter tube-dependent quantities are the electron transmission efficiency $\epsilon$, image magnification in space and time, $m_{x}$ and $m_{t}$, respectively, and electron to light conversion efficiency of phosphor $\xi . \gamma$ is the detector efficiency for either film or CCD readout averaged over P20 phosphor output and includes losses in coupling to the image converter tube. The streak camera response depends on X-ray energy, primarily through the photocathode conversion efficiency $\eta$. Electron signals from the photocathode are due to secondary electrons created by the stopping of energetic electrons produced by photoabsorption in gold. All the parameters except $\eta$ can be combined in a single proportionality constant $\alpha$ that depends only on instrument parameter and not on the X-ray energy. Finally (16) can be written as

$$
S_{x}=\alpha \eta F_{x}
$$

Quantum efficiency $\eta$ for transmission photocathode of gold and CsI has been reported to range from $109 \mathrm{eV}$ to $7.5 \mathrm{keV}$ (Stradling et al 1983). For absolute calibration only the constant $\alpha$ needs to be determined.

\subsection{X-ray emission in presence of magnetic field}

The optical and X-ray streak cameras discussed above have been used in various experiments (Rai et al 1995c, d, 1998; Pant et al 1998). Figure 12 shows the time-dependent $\mathrm{X}$-ray intensity profile emitted from laser-produced plasma in the absence and presence of a magnetic field $(0.6 \mathrm{~T})$. These observations were recorded with a $4 \mu \mathrm{m}$ Zapon filter in front of the photocathode of the X-ray streak camera. It is seen that X-rays are emitted for nearly the same time duration $\tau_{x} \sim 1.5 \mathrm{~ns}$ in both the cases. However the only difference in the intensity profile is that it broadens near the peak when it starts decaying. X-ray emission is maximum between 400 and $500 \mathrm{ps}$ in both cases whereas it increases after the peak in the case when a magnetic field is present as compared to when it is absent. However the total time duration of X-ray emission remains the same. This indicates that an increase in X-ray intensity after $500 \mathrm{ps}$ seems to be due to an increase in plasma density at that time as a result of plasma confinement in the presence of the magnetic field which is in agreement with the results from an X-ray pin-hole camera (figure 19b, see $\S 6.2$ below) which show a decrease in the size of the $\mathrm{X}$-ray emitting plume as a result of magnetic confinement. We can infer from the streak camera measurements that the plasma smoothly expands by nearly 400 to $500 \mathrm{ps}$, after which it starts decelerating due to the presence of the magnetic field, which increases the effective density of the X-ray emitting plume. The increased density and decreased plasma temperature after expansion of $400-500 \mathrm{ps}$ enhances the recombination radiation which seems to be the reason behind increase in the X-ray emission after peak of the intensity profile. The detailed result has already been reported (Rai et al 1999). 

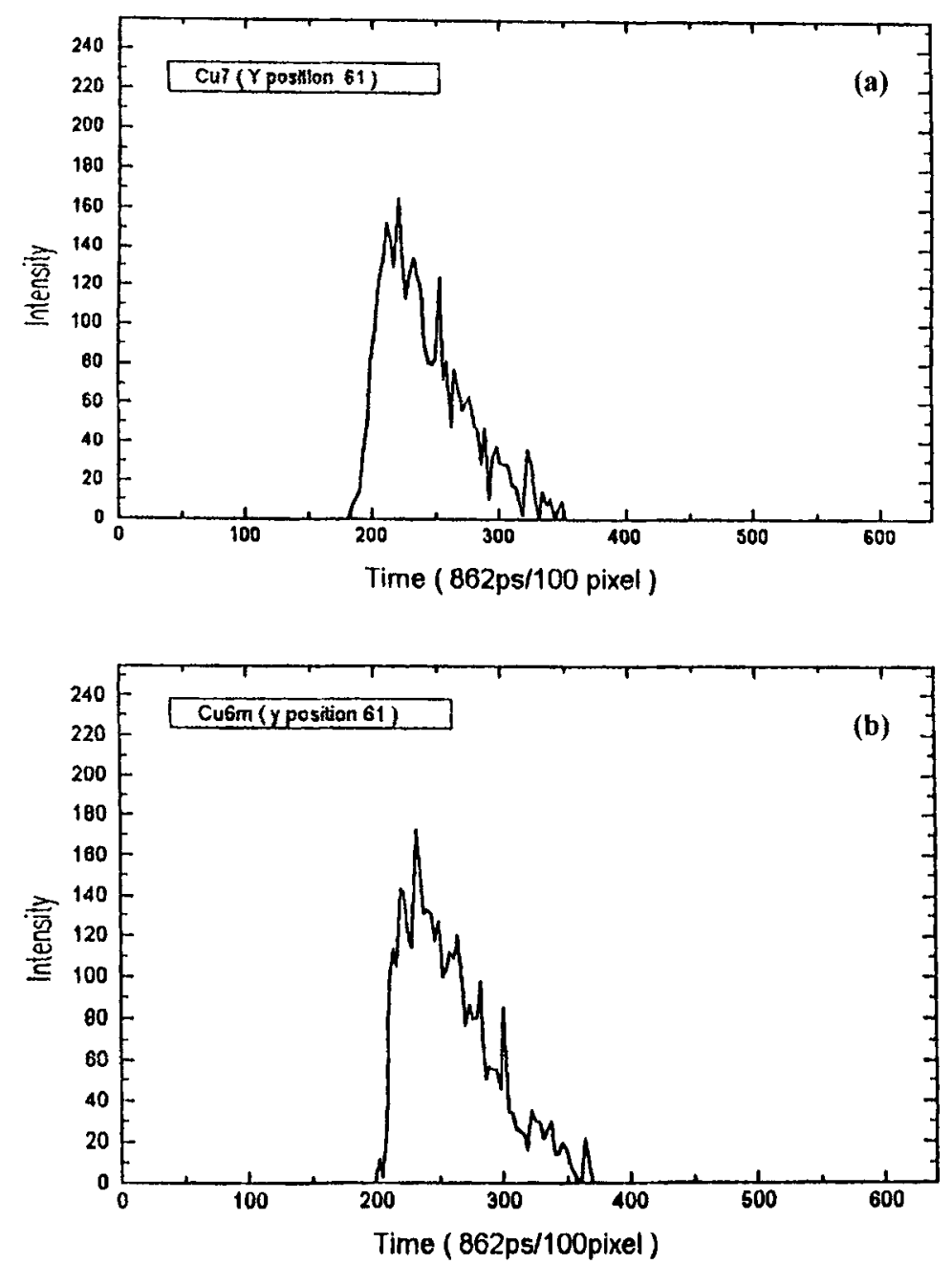

Figure 12. Temporal behavior of X-ray emission in the absence (a) and in the presence (b) of a magnetic field.

\section{X-ray framing camera}

The X-ray framing camera (Stearn et al 1989; Kilkenny 1991; Ze et al 1992) has been useful in capturing several images from a single optical event gated at an adjustable interframe time. The main application of gated X-ray detectors has been in the field of inertial confinement fusion and $\mathrm{X}$-ray laser research. The shutter speed of the framing camera has been decreased to $<50 \mathrm{ps}$ which is necessary to avoid motional blurring during the exposure of moving and $\mathrm{X}$-ray emitting objects. Plasma expansion velocity is found typically $\sim 10^{7} \mathrm{~cm} / \mathrm{s}$ in laser-produced plasma and inertial confinement fusion experiments. If a spatial resolution of $\sim 10 \mu \mathrm{m}$ is required then a shutter time speed of $<100 \mathrm{ps}$ is needed to freeze the motion. There are two methods for gating images, such as the gating of the X-ray image converter tube which is nothing but the streak tube (Shukla et al 1998a, c), and the second is the gating of the proximity-focused microchannel plate detector (Stearn 

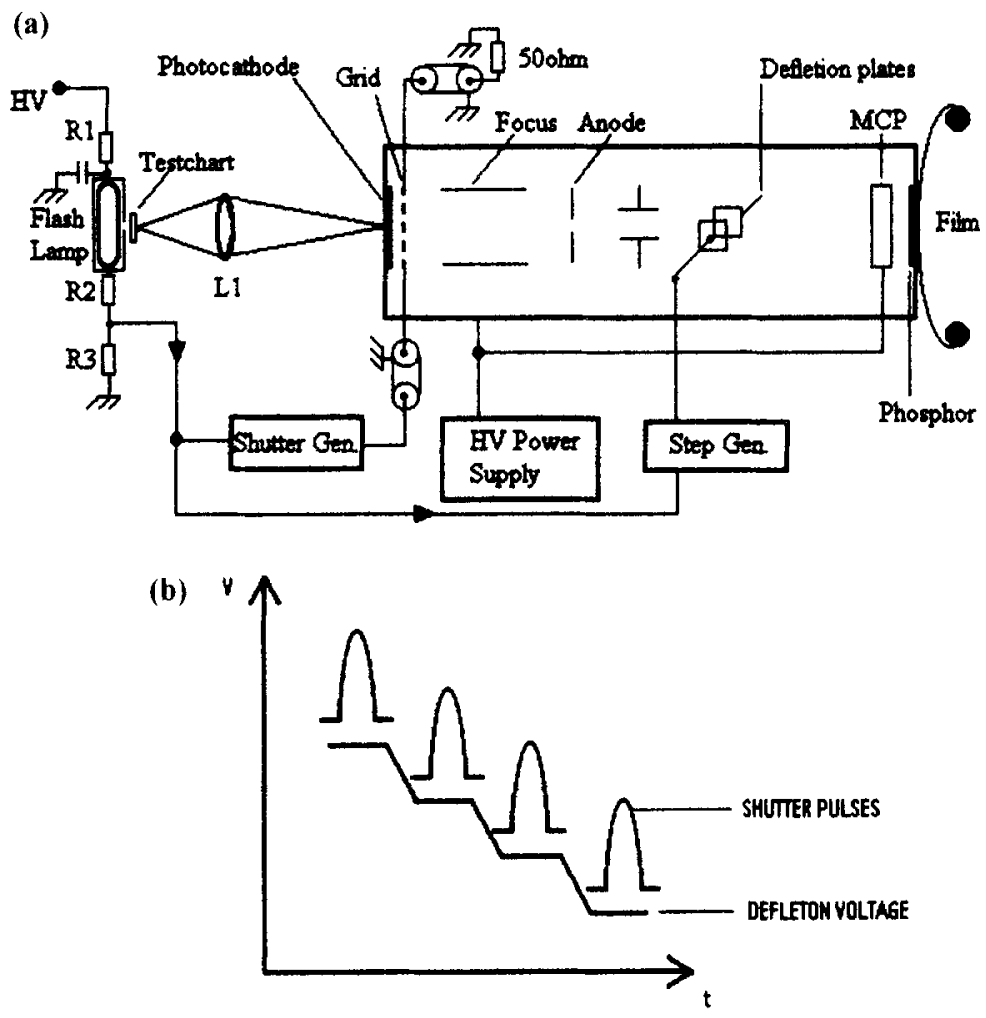

Figure 13. Schematic diagrams of (a) image converter tube for framing camera, and (b) staircase voltage and gate pulse on a synchronized time scale.

et al 1989; Kilkenny 1991). In the first case where the image converter tube (figure 13a) is used for framing the camera, two main changes are required compared to the X-ray streak camera. A small gate pulse of 200 volts and of $3 \mathrm{~ns}$ time duration is applied to the photocathode at a time interval of $5 \mathrm{~ns}$, which allows the photoelectrons to pass through the deflection plates (figure 13a). These photoelectrons create an image on the phosphor screen after proper deflection. A staircase type of voltage (figure 13b) is applied on the deflection plate instead of a fast rising pulse as is required in the case of the streak camera. During the rising part of the staircase voltage the location of the image shifts on the phosphor screen whereas during the flat portion the image is recorded. The main problem with this technique is the loss of spatial resolution at short exposure time, mainly in the direction of deflection. It also has limitations in providing a large number of frames with better time and space resolution.

Nowadays, the second technique (Kilkenny 1991) is being used which has overcome all the shortcomings of the previous techniques. It consists of a microchannel plate coupled with a phosphor screen. There are gold-coated low impedance strip transmission lines on the front surface of the MCP, whereas the opposite side has a plane conducting surface at ground potential. The gold-coated transmission line has three designs out of which the $S$ shaped transmission line on the MCP front is mainly used owing to less complexity in its electronics. A very short $(<100 \mathrm{ps})$ time duration pulse propagates along this transmission line and activates different locations of the transmission line at a certain time interval depending on the propagation velocity of the pulse on the transmission line. The X-ray 
emitting source is imaged on to this transmission line using an array of multiple pin-holes in between the source and the MCP. In this process, more than 10 images can be captured at a time separation decided by the spatial separation of images on the MCP and the speed of pulse propagation through the transmission line. Some work on the testing of first type of framing camera has been performed in collaboration with General Physics Institute Moscow. However development of second type of framing camera is still in progress in our laboratory.

\section{X-ray transmission grating spectrometer}

Generally reflecting (grazing incidence) and transmitting (normal incidence) by crystals and diffraction gratings are widely used (Huddlestone \& Leonard 1965) as dispersing elements for $\mathrm{X}$-ray radiation investigation. Crystal spectrographs are of high resolution $\left(\lambda / \Delta \lambda \sim 10^{5}\right)$, but radiation visibility is low. Therefore, such devices are preferred for the investigation of linear radiation where resolution of thin structures and determination of small spectral shifts are needed. The working range of a crystal spectrograph is limited by $\lambda \sim 28 \AA$ due to the interlayer crystal distance. A diffraction reflection grating operating in the grazing incidence regime provides a wide spectrum of visibility from 10-15 to a few $100 \AA$ with spectral resolution $\sim 10^{2}-10^{3}$. However, they suffer from a complicated alignment and astigmatism problem. However transmission gratings (Alexandrov et al 1988, 1989; Pina et al 1991; Bolotin et al 1993) are of interest as X-ray optics elements. Spectrometers based on such gratings provide a wide working range from a few angstroms to a few $100 \AA$ and good spectrum visibility at a resolution of $\lambda / \Delta \lambda \sim 10^{2}$. In this case alignment is very easy and it can provide spatial resolution throughout the spectral range if a slit or a pin-hole is mounted in front of it. X-ray transmission grating spectrograph (Alexandrov et al 1988, 1989; Pina et al 1991; Schwanda \& Eidmann 1992; Bolotin et al 1993) has proved to be a good tool for determining the radiation temperature of laser irradiated gold cavities along with the spatial and absolute spectral information. A transmission grating spectrometer has been developed (Shukla et al 1998b) to study the spectral property of X-rays being emitted from the plasma as well as to measure the black body radiation temperature from the gold cavity or hohlraum target. It can provide temperature information at various spatial locations of the gold cavity in a single shot of the laser.

\subsection{Design of spectrometer}

The spectrometer works without any optical focussing element. Free-standing gold bars from the grating which is sandwiched between two apertures of the same size. These apertures may be of long slit type $(100 \mu \mathrm{m} \times 3 \mathrm{~mm})$ or of pin-hole $(50 \mu \mathrm{m})$ type. The limit of the wavelength resolution $\Delta \lambda$ is given by the number $n$ of the grating bars within the aperture $(\Delta \lambda / \lambda \approx 1 / n)$. This optimum resolution, however, is only achieved for a point source at a large distance from the grating ( $\gg A^{2} / \lambda$, where $A$ is the slit width). For smaller distances and extended sources, wavelength resolution is determined by geometrical optics

$$
\Delta \lambda=d \cdot S / L,
$$

where $d$ is the grating constant, $S$ the source dimension and $L$ the distance between source and grating. However, this expression is valid only for not too large a slit width

$$
A \ll(R-L) S / R
$$




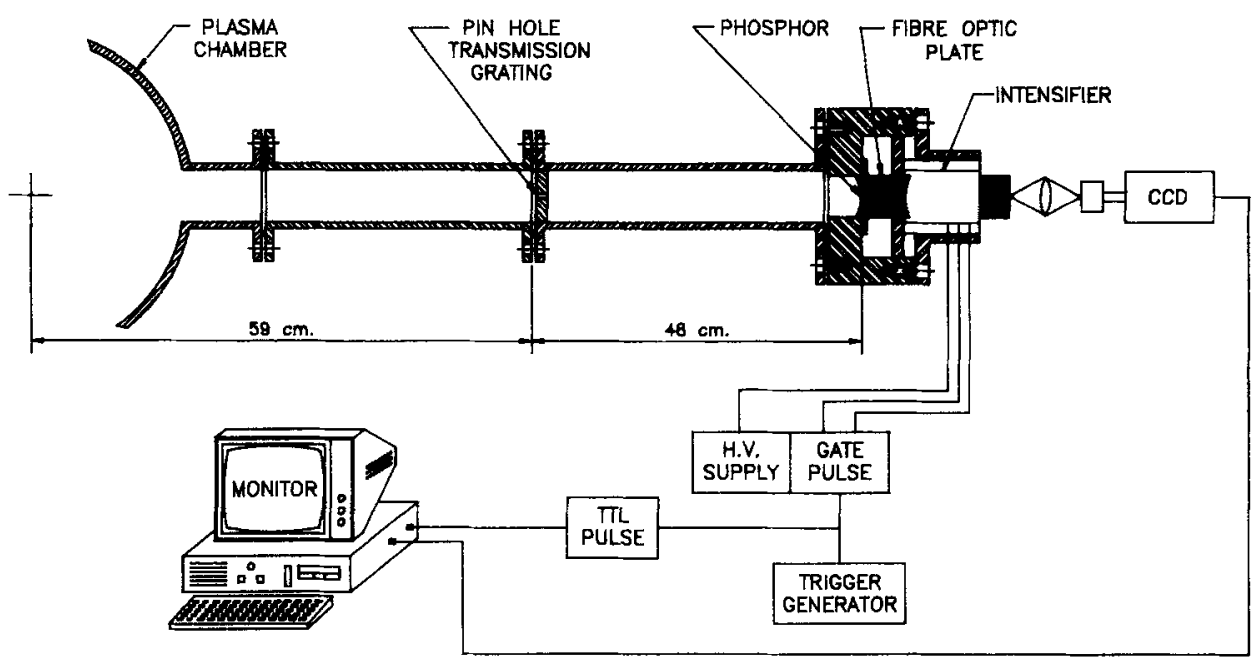

Figure 14. Schematic diagram of the transmission grating spectrometer.

where $R$ is the distance between source and detector. A generalized expression for spectral resolution can be given as

$$
\Delta \lambda=d[(S+A) / L+A / D]
$$

Figure 14 shows a schematic diagram of the transmission grating spectrometer. It consists of a transmission grating of period $d=1 \mu \mathrm{m}(1000$ lines $/ \mathrm{mm})$ placed in between two stainless steel pipes (dia $\approx 50 \mathrm{~mm}$ ) connected to the plasma chamber and a detector consisting of a combination of phosphor screen and image intensifier. The distance between plasma source and grating is $L=55 \mathrm{~cm}$ and between grating and detector is $D=60 \mathrm{~cm}$. The first-order spectrum is most intense compared to those of other orders (other than zeroth order) owing to the largest grating efficiency $(\eta \approx 7 \%)$ for first order. The inverse linear dispersion of the spectrometer can be written as

$$
\mathrm{d} \lambda / \mathrm{d} y=m D / d \cong 16 \AA / \mathrm{mm},
$$

where $m=1$ is for the first order and $y$ is in the direction of dispersion in the plane of the phosphor screen. The limit of spectral resolution is calculated from the geometrical optics relation as

$$
\Delta \lambda=d[(S+A) / L+A / D] \approx 6 \AA
$$

where $S$ is the source dimension $\approx 100 \mu \mathrm{m}$ and $A \approx 100 \mu \mathrm{m}$ is the width of the slit before the grating. The final image on the phosphor screen of the image intensifier is recorded with the help of a CCD camera and a frame grabber.

\subsection{Design of detector system}

The detector of this spectrometer is a phosphor screen coupled to an image intensifier as shown in figure 14. The image intensifier tube consists of a photocathode, antidistortion electrode, microchannel plate (MCP) and phosphor screen that requires high voltage (- 7.5 , $-7.5-5 \mathrm{kV}$ and ground respectively) and low current power supply for its operation. The 




Figure 15. A gate pulse generator for driving the image intensifier tube.

power supply used here is the same as for the streak camera (figure 8) but with a proper divider. The background noise on the phosphor screen of the image intensifier is reduced by applying a gate pulse at the input terminal of the MCP present in the tube generated by a gate pulse generator as shown in figure 14. It consists of an oscillator of SMPS as shown in figure 8. The secondary of the transformer consists of 560 turns (SWG28 copper wire). The variable resistance at the common base of the transistors BUL45 varies the voltage in this supply. The gain of the MCP can be changed on varying the field between its two terminals by applying a pulse of amplitude variable from -100 volts to $-1 \mathrm{kV}$ and a few milliseconds time duration at the $\mathrm{MCP}_{\text {input }}$ of the intensifier. Triggering high voltage transistor BU208A at its base by a monostable multivibrator designed using 2N2219 transistors as shown in figure 15 generates this pulse.

\subsection{Testing of spectrometer}

The spectrometer described above was tested by recording the X-ray emission from copper and gold laser-produced plasma. An Nd: YAG laser (75 mJ and $35 \mathrm{ps}$ ) was focused to $100 \mu \mathrm{m}$ spot size by a lens (focal length $=30 \mathrm{~cm}$ ) on copper and gold planar targets kept in vacuum of $10^{-2}$ Torr. The spectrum from the phosphor screen was recorded with the help of a CCD camera and was processed by the processing software PROMISE (Vora et al 1994). Figures 16 and 17 show the intensity profiles of X-ray spectra obtained from copper and gold plasma respectively. The X-ray spectra of copper and gold plasmas were found to be in good agreement with other reported results (Schwanda \& Eidmann 1992; Bolotin et al 1993). In the case of copper, the dominant line spectra from $L$ shell at nearly $10 \AA$ provides clear single peaks of both first and second order, whereas in the case of gold plasma, which 


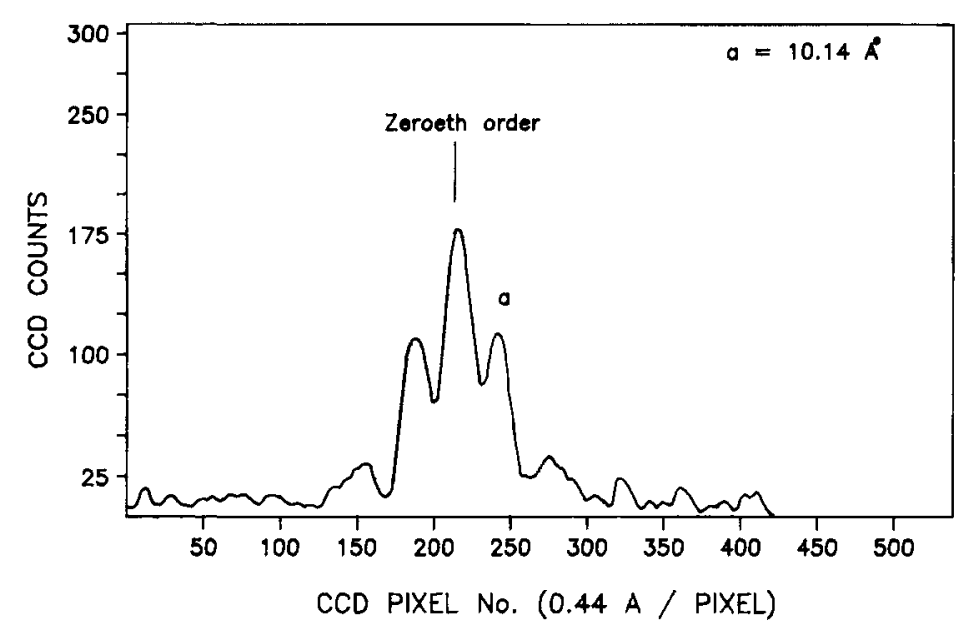

Figure 16. Intensity profile of $X$-ray spectrum from copper plasma.

is partially ionized, emission comes from the transitions in the outer shells $(M, N$ and $O$ shell). It provides broad band emission (Sigel et al 1987) which seems to be due to the combination of line and continuum spectra. Probably due to broad band emission from gold, first and second orders are not clearly resolvable from these spectra. However, deconvolution of raw spectra is needed to get an absolute spectra. The process of deconvolution of spectra either from the phosphor screen or from X-ray film is in progress.

\section{X-ray pin-hole camera}

An X-ray pin-hole camera (Jacob et al 1976; Radziemski \& Cramers 1989) is used to image the X-ray emitting plume of laser-produced plasma onto an image plane. Functioning of the pin-hole camera follows the principle of geometrical optics. The image becomes more distinct as the pin-hole is made smaller. But it should not be so small that diffraction becomes significant and affects the spatial resolution of the camera. For a small pin-hole,

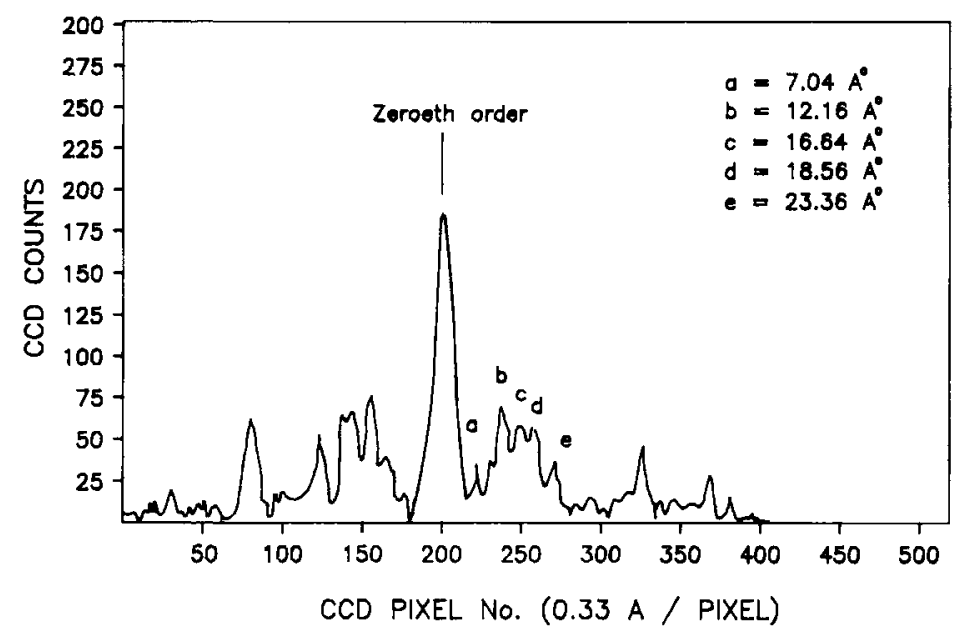

Figure 17. Intensity profile of X-ray spectrum from gold plasma. 
Raleigh criterion are used where a point forms a diffraction pattern in such a way that first minimum of one pattern coincides with the first minimum of the second. The point will be at the limit of resolution defined by

$$
\theta=1.22 \lambda / a,
$$

where $\lambda$ is the wavelength of light and $a$ is the size of the pin-hole. For getting optimum resolution the following relation should be satisfied

$$
1 / d+1 / f=4 \lambda / a^{2}
$$

where $d$ is the distance between object and pin-hole, $f$ is the separation between pin-hole and image plane. In a real sense, a pin-hole camera acts as a lens of focal length

$$
f=a^{2} / 4 \lambda \text {. }
$$

The spatial resolution of the pin-hole camera is given by

$$
\Delta s=d \theta=d(5 / 6)(a / f)[((d / f)+1) /(d / f)], \quad \text { For } a \gg \lambda,
$$

where $f / d$ is the magnification of the image. In simple terms spatial resolution can be given as

$$
\Delta s=a[(M+1) / M]
$$

which in fact is nearly equal to the size of the pin-hole. The X-ray pin-hole camera (Jacob et al 1976; Attwood 1978; Lindl 1995) is one of the important diagnostics used to study the symmetry of implosion in direct and indirect drive inertial confinement fusion experiments. It provides time-integrated spatial information about $\mathrm{X}$-ray emission from planar or microballoon targets.

\subsection{Design of the pin-hole camera}

A phosphor screen and intensifier based pin-hole camera (Rai et al 1996b) has been developed (figure 18a) to study the spatial characteristics of X-ray emitting plasma plumes. The pin-hole camera consists of two stainless steel tubes, one sliding inside the other. One free end of the inner tube has a pin-hole of $30 \mu \mathrm{m}$ diameter, whereas the free end of the outer tube has a phosphor screen (P11 phosphor coated on a fibre optic plate). An image intensifier tube is attached to intensify the image. Magnification of the camera can vary from 2 to 10 . The image is initially recorded on a P-11 phosphor screen coated on a fibre optic plate, which is also developed in CAT. This image is intensified using a gated image intensifier tube and recorded using a CCD camera. The X-ray detector (combination of phosphor screen, intensifier and its driving electronics) in the pin-hole camera is similar to that described in $\S 5$. Spatial resolution of this system is estimated to be $\sim 30 \mu \mathrm{m}$ but is actually $\sim 50 \mu \mathrm{m}$ and is limited by the spatial resolution of the image intensifier tube. Pinhole cameras having film detection as well as MCP detection system have also been developed in our laboratory (Prasad et al 1998). They are reported to have better spatial resolution, $\sim 10 \mu \mathrm{m}$. The phosphor screen and intensifier-based pin-hole camera has two advantages over the film or MCP recording system. It can provide on-line information about the picture and can be operated under bad vacuum conditions $\left(10^{-2}\right.$ to $10^{-3}$ torr $)$ without any problem whereas MCP detection needs vacuum of the order of $10^{-5}$ torr. Pin-hole pictures were processed using the software PROMISE (Vora et al 1994) developed 


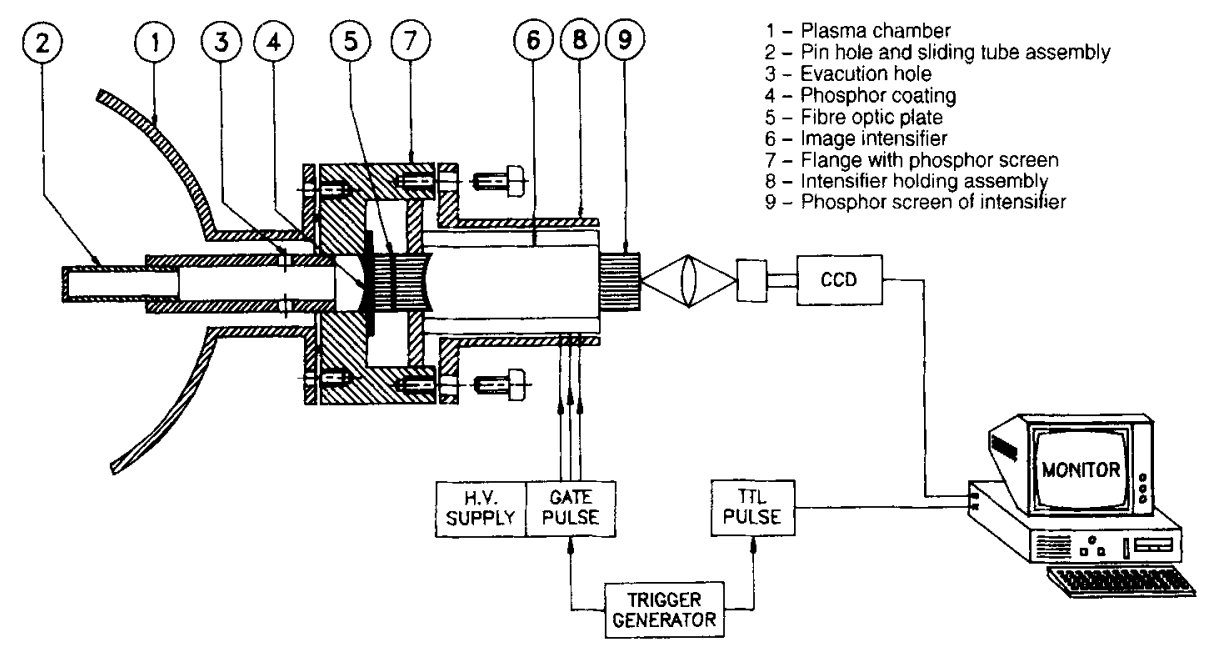

Figure 18. Schematic diagram of X-ray pin-hole camera.

in CAT. An appropriate detection system can be chosen depending on the requirement of the experiment. This camera has been used to record the dimensions of X-ray emitting plumes of laser-produced plasma expanding across the magnetic field as presented in the following section.

\subsection{Study of plasma plume}

The pin-hole camera developed in our laboratory has been used to study the X-ray emission from laser-produced plasma expanding across the magnetic field (Pant et al 1998; Rai 1998). Figure $19 \mathrm{~b}$ shows the digitized image of time-integrated $X$-ray pin-hole pictures of

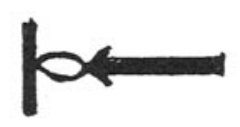

(a)

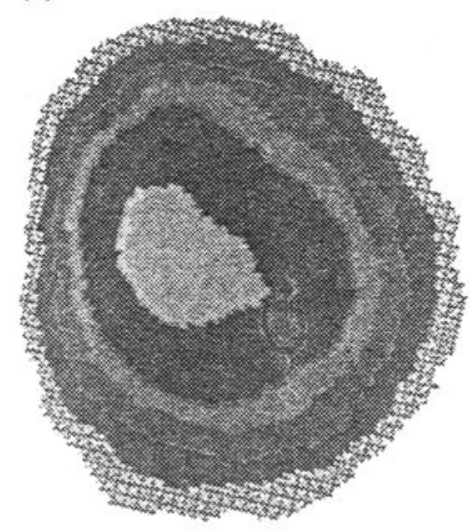

(b)

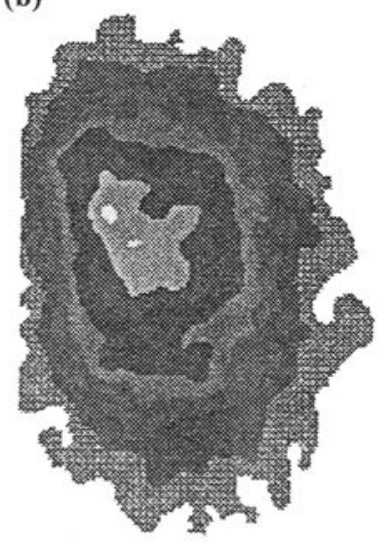

Figure 19. Time-integrated pin-hole image of X-ray emitting plume expanding in vacuum (a) and across a uniform magnetic field (b). 
laser-produced plasma expanding in vacuum with and without the magnetic field. Expansion of the plasma without the magnetic field is nearly spherical in nature. The spatial extent of the $\mathrm{X}$-ray emission region is measured up to $\sim 450 \mu \mathrm{m}$ from the target surface. The hottest portion of the plasma (highest brightness spot) in the centre ranges from $\sim 100 \mu \mathrm{m}$ to $200 \mu \mathrm{m}$ (figure 17b), but when the plasma is allowed to expand across a $0.6 \mathrm{~T}$ magnetic field it shows distortion at each isointensity contour as is shown in figure $17 \mathrm{~b}$. Distortion in the smooth expansion of the plasma boundary seems to be due to generation of some kinds of instabilities as a result of deceleration in plasma expansion in the presence of the magnetic field. Effect of the magnetic field in the form of plasma distortion can be seen even in the hottest portion. In this experiment, ionic and electron larmor radii are $\sim 3$ and $0.05 \mathrm{~mm}$ respectively. Ion larmor radius was larger than the density gradient scale length $C_{s} \tau \sim 2-3 \mu \mathrm{m}$, which shows that ions are effectively unmagnetized in comparison to electrons. In this condition, generation of Rayleigh-Taylor or finite larmor radius instabilities are expected as reported earlier (Ripin et al 1987). However the presence of KelvinHelmholtz instability due to electron velocity shear (as a result of combined effect of selfgenerated spontaneous magnetic field and external magnetic field) cannot be ruled out. The spatial extent of X-ray emission from the target surface in the presence of magnetic field is limited to $\sim 350 \mu \mathrm{m}$ across the magnetic field. However no significant change was found in plasma size along the magnetic field. These pictures indicate that the plasma is being confined in the presence of the magnetic field, which is expected. An increase in X-ray emission is expected due to the increase in plasma density as a result of decrease in plasma expansion velocity because $\mathrm{X}$-ray intensity due to Bremhsstrahlung, recombination or line radiation, are all directly proportional to the square of plasma density. X-ray intensity is enhanced 2-3 times in this condition (Rai et al 1998; Pant et al 1998). This enhancement as well as its temporal development has been confirmed by X-ray streak camera records (figures $12 \mathrm{a}$ and $\mathrm{b}$ ).

\section{PVDF sensors}

Highly active piezoelectric polymers (Coufal et al 1987; Chen \& Payne 1995; Obara et al 1995; Boustie et al 1996) have been known for over 25 years. In this period, considerable effort has been made in the study of the structure of these polymers to enhance piezoelectricity as well as long term changes in the piezoelectric and pyroelectric properties of these materials. However, in the last decade people have started using polyvinyledene difluoride (PVDF) polymer as the best-suited for making pyroelectric calorimeters and piezoelectric sensors. These sensors use polyvinyledene difluoride (PVDF) thin sheets having metal (aluminum) coatings on both the sides which are permanently polarized. The sheets act as pyroelectric detectors (Coufal et al 1987; Obara et al 1995) because any change in the temperature difference between the two surfaces generates corresponding change in polarization and, as a result, voltage signals across both electrodes. PVDF sheets act as piezoelectric detectors also (Obara et al 1995; Rai et al 1994b) used to measure the stress generated either mechanically or through laser-induced ablation pressure in laser plasma experiments with planar target geometry.

\subsection{Pyroelectric calorimeter}

Pyroelectric material can be characterized by its pyroelectric coefficients $P(r, t)$ where $r$ and $t$ are spatial and temporal dependence. Any change in the temperature $\Delta T(r, t)$ with 
respect to the reference temperature $T_{0}(r, t)$

$$
\Delta T=T(r, t)-T_{0}\left(r, t_{0}\right),
$$

produces a small change in the polarization of the material. The change in polarization in a PVDF film of thickness $l$ induces an electric charge $Q(t)$ across both sides of the metallized electrodes and is given by

$$
Q(t)=1 / l \int P(r, t) \Delta T(r, t) \mathrm{d} r .
$$

Change in the energy content of the pyroelectric film can be written as

$$
\Delta U(t)=\int \cos T(r, t) \mathrm{d} r
$$

where $c$ is specific heat and $\rho$ is density of the pyroelectric material. If we assume that the material properties are temperature-independent and the material is homogeneous, then the induced charge is proportional to the change in energy content of the pyroelectric film as

$$
Q(t)=[P / c \rho l] \cdot \Delta U(t)
$$

If heat loss from the pyroelectric element into the surroundings is neglected, the element works as a pyroelectric calorimeter. Finally, the signal ( $V$ in volts) obtained from a calorimeter can be given as

$$
V=P l \Delta T / \epsilon .
$$

where $\epsilon$ is the dielectric constant of the PVDF material.

\subsection{Piezoelectric sensor}

Piezofilm thin foil after permanent polarization develops dipoles aligned along the axis of poling, in this case normal to the plane of the piezofilm. Piezoelectric activity is found to be directionally dependent. In fact, these materials are anisotropic, their electrical, mechanical and electromechanical properties differ for electrical or mechanical excitation along different directions denoted as 1,2 and 3 . Notation 3 indicates the direction perpendicular to the plane of the film. The induced signal generated by a piezofilm of thickness $t$ will be given by

$$
E=V_{0} / t=g_{33} X_{3} \text { (volts) }
$$

where $g_{33}$ is the piezofilm constant $(3,3$ mean direction of permanent polarization and direction of stress which is perpendicular to the plane) and $X_{3}$ is the applied stress on the film. It has been reported (Tam 1986) that duration of pressure signal $T_{a}$ is related to the laser pulse duration $T_{L}$ and the shock/acoustic transit time $T_{t}$ is defined as the shock/acoustic propagation time across the pressure source of length $l$ in the direction of observation,

$$
T_{a} \approx\left(T_{L}^{2}+T_{t}^{2}\right)^{1 / 2}
$$

In fact, $l \approx 10^{-5} \mathrm{~cm}$ is a typical laser absorption length and $v$ is the velocity of sound $\approx 10^{6} \mathrm{~cm} / \mathrm{s}$ which gives $T_{t} \sim 10^{-11} \mathrm{~s}$, which shows that $T_{a}$ is of the order of $\sim 10 \mathrm{ps}$. If laser duration is very small, we can get 10 ps duration pressure pulse. 


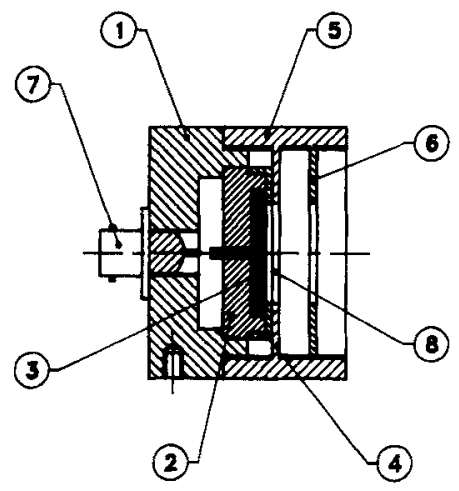
1. S.S BODY
5. SCATTERING PLATE HOLDER
2. TEFLON HOLDER
6. PLATE HOLDING RING
3. COPPER BACK PLATE
7. BNC OUTPUT
4. FOIL HOLDING RING
8. PVDF FOIL

Figure 20. Cross-sectional view of pyroelectric calorimeter.

\subsection{Design of calorimeter and pressure sensor}

Design of the calorimeter consists of a circular backplate of dia $15 \mathrm{~mm}$, which is electrically connected to the central pin of a BNC connector. PVDF film is pressed by a metallic annular disc with a hole in the centre $(\mathrm{dia} \approx 10 \mathrm{~mm})$ and a locking system so that the one side coating of the film has electrical connection with the backplate and the front surface has electrical connection with the body through the annular ring and locking system. A circular hole in between the annular ring and locking system causes the light or radiation to fall on the film. This system acts as a calorimeter if radiation falls on it. It provides an electrical signal proportional to pressure through BNC if stress is applied on the front surface. For laser-induced stress measurement, the target is kept in such a way that the back of the target is in physical contact with the front surface of the thin sheet in the pressure sensor. The signal obtained in the calorimeter mode of operation has slow variation in time (ms) whereas the stress signal has very fast time scales, that is in nanoseconds. A pyroelectric calorimeter (figure 20) has been developed (Rai et al 1994a, c) using PVDF foil to measure the energy of X-rays emitted from laser-produced plasma. It provides two types of signal in the pyroelectric calorimeter mode. One of the signals has fast response, owing to the thermo-elastic effect in the foil, whereas the other has slow response and is actually a time-integrated calorimetric signal. However, both the signals are proportional to the absorbed X-ray energy (Rai et al 1994a) and can be calibrated for absolute measurement. Finally, the sensitivity of the calorimeter is estimated on the basis of (30) and is given as

$$
\Delta V=400 \Delta J
$$

where $\Delta V$ is the induced signal in the calorimeter and $\Delta J$ is the absorbed fraction of laser energy by the calorimeter. This provides a sensitivity of $400 \mathrm{mV} / \mathrm{mJ}$ (absorbed) which is in close agreement with the experimental measurements for the laser of wavelength $\lambda=1.06 \mu \mathrm{m}$. However, during the calibration of this sensor, it must be kept in mind that only $\approx 5 \%$ of the incident laser energy is absorbed and the rest is reflected as a result of the 

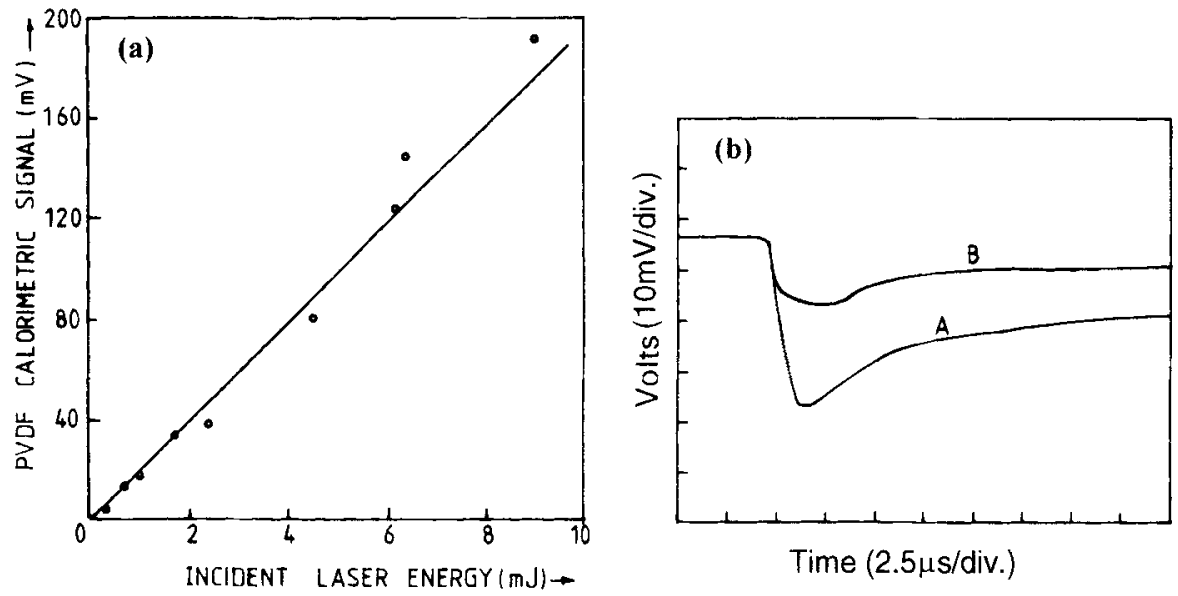

Figure 21. (a) Calibration curve for calorimeter with (Nd: YAG) laser energy. (b) Oscillogram of calorimetric signals obtained from X-ray source.

thin coating of aluminum on the sensor. Figure 21 a shows the calibration curve of this calorimeter as a function of incident laser energy.

This detector has been used as a calorimeter for X-ray radiation with the assumption that all the incident $\mathrm{X}$-rays falling on the sensor are absorbed by the aluminum coating and are utilized for signal generation. However, sensitivity of the calorimeter may increase in the case of X-ray detection if gold is coated (presently not available) on the absorbing surface whereas black coating on the front electrode enhances its sensitivity many-fold for the spectral range UV, Vis, IR. Figure $21 \mathrm{~b}$ shows the oscillogram of calorimetric signals obtained by detecting $X$-rays from gold and aluminum plasma. Here one can observe that amplitude is larger in the case of X-rays emitted from aluminum plasma (A) in comparison to the gold plasma (B). This observation is in contradiction with reported results because gold conversion efficiency is greater than aluminum. But the reason behind this observation however is simple. As the gold strip is attached on the aluminum target in this experiment, signals due to gold plasma are recorded when the laser beam falls normally on the target, whereas X-rays from aluminum target are detected by rotating the aluminum plate such that the incident laser falls at a small angle to the target normal $\left(\approx 20-30^{\circ}\right)$. In this case, increase in signal is expected due to resonance absorption of laser light at oblique incidence (Rai et al 1996a). Hence, large amplitude is recorded in the case of aluminum plasma (figure $21 \mathrm{~b}$ ).

This sensor is attached at the back of the thick planar metal target to measure the stress generated during laser beam interaction with the target. A bipolar electrical signal is recorded when the laser beam intensity does not produce plasma, whereas signals become unipolar for laser intensity generating plasma. Bipolar signals are the result of compression and rarefaction waves propagating in the material in the form of thermoelastic waves whereas unipolar signals are due to compression waves generated by the laser-induced shock. Figure 22a shows bipolar signals generated due to interaction of laser light with a stainless steel (S.S.) plate of $0.5 \mathrm{~mm}$ thickness. There are many echo pulses, denoted by $A_{1}, A_{2}$ and $A_{3}$, of the main compressional pulse $A$. The delay between $A$ and $A_{1} 175.5 \mathrm{~ns}$ provides the speed of sound in S.S. as $5698 \mathrm{~m} / \mathrm{s}$. This is one of the applications of this technique and this can be used to determine the thickness of sample materials (Tam 1986). 
This sensor is used to find the scaling of ablation pressure generated in aluminum and copper target with incident laser intensity (figure 22b) (Rai et al 1994c),

$$
P_{a} \propto\left(I_{L}\right)^{\alpha}
$$

where $\alpha=0.85$ is in close agreement with the value $0.6-0.8$ reported (Salzmann et al 1983). In another experiment, it was found that for constant laser intensity, ablation pressure increases with an increase in ambient chamber pressure. This observation is in agreement with the reported results

$$
P_{a}=2 I / v
$$

where $I$ is the laser intensity and $v$ is the plasma expansion velocity which shows small decrease with increase in ambient chamber pressure, probably due to pressure confinement of plasma and consequent increase in ablation pressure.

\section{Optical shadowgraphy}

Optical and X-ray backlighting diagnostics play an important role in the study of the hydrodynamics of laser-produced plasma in planar or spherical target experiments (Jacob et al 1976; Attwood 1978). We have used optical shadowgraphy diagnostics (Desai et al 1996) to study the dynamics of thin gold-seeded plastic targets. A part of the main laser beam Nd: YAG $(35 \mathrm{ps}, 75 \mathrm{~mJ})$ operating at $\lambda \sim 1.06 \mu \mathrm{m}$ is split, converted into second harmonics $(\lambda=0.53 \mu \mathrm{m})$ and used for backlighting the target whereas the main beam of the laser is used to irradiate the thin foil target. Second harmonic beam is optically delayed according to the requirement of the experiment and the foil target is imaged at a distance of $\sim 75 \mathrm{~cm}$ such that it provides magnification of $\approx 10$. The time duration of the probe beam decides the speed of the technique. The shadow of the accelerated thin foil target is recorded using a polaroid film as well as a CCD camera. This technique is used to study gold-seeded plastic targets. It has been shown that Rayleigh-Taylor ( $R-T)$ instability is suppressed in the presence of seeded particles.

\section{Other important diagnostics}

In addition to these diagnostics, a K-edge spectrometer was also developed which has a four-pin photodiode as a detector. However, software is being developed to determine the on-line temperature of plasma. In addition to these, there are some other important diagnostics which are required to study hot plasma or the burn history of fusion plasma such as neutronics, X-ray framing cameras, crystal spectrometers and zone plate imaging systems, as well as neutron streak cameras. Some of these diagnostics are under development whereas some are still at the planning stage.

\section{Future applications of these diagnostics in fusion studies}

Some of the diagnostics discussed above have been used in one of our experiments for the study of laser-produced plasma expanding across a magnetic field (Rai et al 1998a; Pant et al 1998). We observe that magnetic confinement of laser-produced plasma generates many effects out of which enhancement in X-ray emission and decrease in expansion velocity may be applicable in many other ways. In the light of these results we can say that 

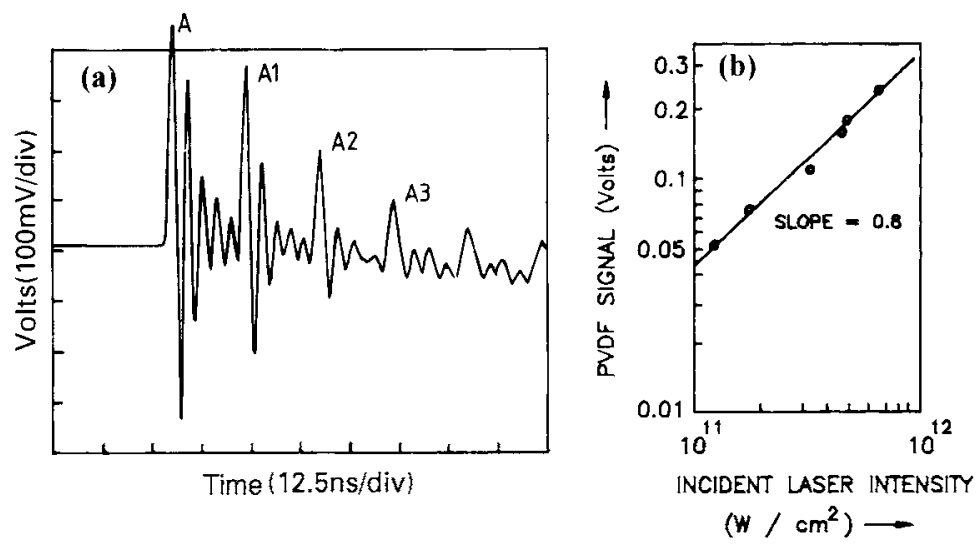

Figure 22. (a) Oscillogram of compressional wave in SS plate $(0.5 \mathrm{~mm})$. (b) Variation of PVDF signal (amplitude of stress wave) with laser intensity.

the presence of a magnetic field along the entrance hole of hohlraum may enhance the Xray emission in the hohlraum cavity and, as a result, the compression of the microballoon capsule, which will increase pellet gain during an indirect drive inertial confinement fusion (ICF) experiment. Even the enhancement in X-ray emission in the presence of high chamber pressure indicates that these results may be applicable in increasing pellet gain during the gas-filled hohlraum experiment (Delamater et al 1996). However another experimental result (Rai et al 1998b) shows that some high energy ions are also generated in the presence of the magnetic field, which may create a loss in plasma energy. This indicates that a balance must be established to retain the advantage of using magnetic fields in ICF experiments.

Illumination uniformity of microballoon pellets in hohlraum cavities (Lindl 1995) depends mainly on the geometrical management of the emission surface of the X-ray. The location of the X-ray emitter and the incident angle of the laser light to the emitter are important parameters for obtaining uniform illumination. Detailed characterization of hohlraum targets such as radiation confinement and time-dependent spectral and temperature measurements are the main areas, where chiefly transmission gratings, streak and pinhole cameras are applicable.

$\mathrm{X}$-ray imaging diagnostics are indispensable for investigating the structure of the plasma and the stability of implosion (Nakai 1990; McCrory 1993). Time-resolved X-ray photography employing X-ray streak cameras and/or X-ray framing (Stearns et al 1989) cameras will be applicable to investigate the hydrodynamics of the implosion process. X-ray shadowgraphy with separate laser-produced plasma as X-ray source is a useful tool to diagnose implosion dynamics as well as RT instability in the acceleration and stagnation phase of the target (Kilkenny et al 1994).

Study of generation and dynamics of shock as a result of laser-matter interaction along with equation of state study using optical streak cameras can provide information for further improvement in the design of microballoon targets for better pellet gain. Good quality laser-produced shocks are generated by both direct as well as indirect drive methods (Benuzzi et al 1996; Batani et al 1996).

In fact there are many planar target experiments that can provide better insight into the physical processes taking place during laser plasma interaction which may be relevant to inertial confinement fusion experiments. 


\section{Conclusion}

In summary, we have reviewed the development of some of the important diagnostics useful for laser plasma interaction as well as fusion studies. Out of these, some diagnostics are complicated while others are not, but all of them have their own importance. A wide variety of plasma parameters can be obtained using these diagnostics during planar as well as microballoon or hohlraum target experiments. However, these diagnostics are useful not only for these experiments but also have wide applicability in other fields of research as well. Transient X-ray and optical emissions from various other sources such as exploding wire experiments, dense plasma focus (DPF) experiments and magnetic confinement fusion machine Tokamak etc. also need such high speed X-ray diagnostics. The pressure sensor can be used in the study of equation of state under high pressure. These pressures may be generated either mechanically or by laser-produced plasma. Some of these diagnostics are simple in design and can be made easily in any laboratory for experiments in any related field.

Authors are grateful to Y B S R Prasad, T Desai, A Upadhyay, P K Tripathi, V K Senecha and R K Khardekar for help and cooperation in the above work. Helpful discussions with V K Chevokin of General Physics Institute Moscow, A A Rupasov and A S Shikanov of P N Lebedev Physics Institute, Moscow and R Arya of the laser instrumentation group are also gratefully acknowledged.

\section{References}

Adams F P, Ng A, Gazit Y 1987 Simple high-speed X-ray diode. Rev. Sci. Instrum. 58: 1130-1132 Alcock A J, Richardson M C, Schelev M Ya 1970 The application of laser triggered spark gaps to electro-optical image converter camera. Proc. IX Int. Congress on High Speed Photography (New York: SMPTE) pp 192-197

Alexandrov Yu M, Koshevoi M O, Murashova V A, Nikitina T F, Rupasov A A, Sklizkov G V, Shikanov A S, Yakimenko M N, Zakharenkov Yu A, Eidmann K, Sigel R, Tasakiris G D 1988 $\mathrm{X}$-ray spectrometer using a free standing transmission grating and a microchannel plate as detector for laser plasma studies. Laser Particle Beam 6: 561-567

Alexandrov Yu M, Koshevoi M O, Murashova V A, Nikitina T F, Rupasov A A, Shikanov A S, Sklizkov G V, Yakimenko M N, Zakharenko Yu A, Eidmann K, Sigel R, Tsakiris G D 1989 Fourchannel X-ray spectrometer on a transmission grating with a combined system of detection. Rev. Sci. Instrum. 60: 2247-2248

Armentraut C J, Geddes J B, Lee P, Canfield L R 1988 UV to soft X-ray survey using a compact biplanar diode array. Rev. Sci. Instrum. 59: 1843-1845

Attwood D T 1978 Diagnostics for the laser fusion program: Plasma physics on the scale of microns and picoseconds. IEEE J. Quantum Electron. QE-14: 909-923

Baker R J 1991 High voltage pulse generation using current mode second breakdown in a bipolar junction transistor. Rev. Sci. Instrum. 62: 1031-1036

Batani D, Bossi S, Benuzzi A, Koening M, Faral B, Boudenne J M, Grandjouan N, Atzeni S, Temporal M 1996 Optical smoothing for shock wave generation: Application to the measurement of equation of state. Laser Particle Beam 14: 211-223

Beck G 1976 Photodiode and holder with 60 ps response time. Rev. Sci. Instrum. 47: 849-853

Benuzzi A, Lower T, Koening M, Faral B, Batani D, Beretta D, Danson C, Pepler D 1996 Indirect and direct laser driven shock waves and application to copper equation of state measurement in the 10-40 Mbar pressure range. Phys. Rev. E54: 2162-2165 
Bradley D J, Loddy B, Sibbet W. Sleat W E 1972 Picosecond electron optical chronography. Appl. Phys. Lett. 20: 219-221

Bolotin V A, Burdonsky I N, Velikovich A L, Gavrilov V V, Golbery S M, Zhuzhukalo E V. JavJalets S V, Kondrashov V N, Kovalsky N G, Pergament M I, Koshevoi M O, Rupasov A A, Shikanov A S 1993 Experimental testing of thin-shell stable acceleration for ICF schemes with direct and indirect drive. Laser Particle Beam 11: 127-135

Boustie M, Couturier S, Romain J P, Zagouri D, Simonet H 1996 Shock pressure and free surface velocity measurements in confined interaction: Response of new $\mathrm{VF}_{2} / \mathrm{VF}_{3}$ piezoelectric gauges. Laser Particle Beam 14: 171-179

Cairn R E. Sanderson J J 1980 Laser plasma interaction (Edinburgh: Inst. of Physics Publishing)

Campillo A J, Shapiro S L 1983 Picosecond streak camera fluorometry - A review. IEEE J. Quantum Electron. QE-19: 585-602

Chen Q X, Payne P A 1995 Industrial applications of piezoelectric polymer transducers. Meas. Sci. Technol. 6: 249-267

Coufal H J, Grygier R K, Horne D E. Fromm J E 1987 Pyroelectric calorimeter for photo thermal studies of thin films and absorbates. J. Vac. Sci. Technol. A5: 2875-2889

Cunin B, Mieche J A. Sipp B, Schelev M Ya, Serduchenko J N, Thebault J 1980 Sweep devices for picosecond image converter streak camera. Rev. Sci. Instrum. 51: 103-110

Delamater N D et al 1996 Symmetry experiments in gas filled hohlraum at NOVA. Phys. Plasma 3: 2022-2028

Desai T, Asthana M. Shukla M. Rai V N 1996 Suppression of hydrodynamic instabilities in laser ablated thin solid target: An experimental study. Sixteenth IAEA Fusion Energy Conference. Montreal, Canada

Guide V, Novokhatsky AV 1995 A proposal for a radio frequency based streak camera with time resolution less than $100 \mathrm{fs}$. Meas. Sci. Technol. 6: 1555-1556

Hooper M B, Osborne P 1995 Inertial confinement fusion (Edinburgh: Inst. of Physics Publishing) Huddlestone R H. Leonard S L 1965 Plasma diagnostic techniques (New York: Academic Press)

Jacob S F. Scully M O, Sergent III. Cantrell C D III 1976 Laser induced fusion and X-ray laser studies (New York: Addison-Wesley)

Kilkenny J D 1991 High speed proximity focused X-ray cameras. Laser Particle Beam 9: 49-69

Kilkenny J D, Glendinning S G, Haan S W, Hammel B A, Lindl J D, Munro D, Ramington B A, Weber S V, Knauer J P, Verdon C P 1994 A review of the ablative stabilization of the RayleighTaylor instability in regimes relevant to inertial confinement fusion. Phvs. Plasmas 1: 1379-1389

Kinoshita K, Inuzuka E, Takiguchi Y, Okada H, Suzuki K, Hayashi I. Tsuchiya Y, Oba K, Medecki H, Stradling G L 1983 Demountable photocathode X-ray streak camera. Proc. SPIE 427: 36-44

Kinoshita K, Ito M, Suzuki Y 1987 Femtosecond streak tube. Rev. Sci. Instrum. 58: 932-938

Kruer W L 1988 The physics of laser plasma interaction (New York: Addison-Wesley)

Lee P, Gerhard J. Armentraut C J, Snider R T 1988 Vacuum ultraviolet and ultrasoft X-ray diode detectors for tokamak plasma. Rev: Sci. Instrum. 59: 883-887

Lindl J 1995 Development of indirect drive approach to inertial confmement fusion and the target physics basis for ignition and gain. Phys. Plasmas 2: 3933-4024

Max C E 1982 Laser plasma interaction (eds) R Balian. J C Adams (Amsterdam: North Holland) (see also: Theory of coronal plasma in laser fusion targets. UCRL-53107 (1982), Report of Lawrence Livermore National Lahoratory, California)

McCrory R L $i 993$ Laser driven IC.F experiments. Nuclear fusion by inertial confinement: A comprehensive treatise (eds) Guellermo Velarde. Yagal Ronen, J M Martinez (Boca Raton, FL: CRC Press) pp 555-596

Nakai S 1990 Inertial confinement fusion: A technical aspect. Nucl. Fusion 30: 1863-1878

Obara T, Boume N K, Mebar Y 1995 The construction and calibration of an inexpensive PVDF stress gauge for fast pressure measurements. Meas. Sci. Technol. 6: 345-348

Pant H C, Rai V N, Shukla M 1998 Behavior of expanding laser produced plasma in a magnetic field. Phys. Scr. T75: 104-111 
Pina L, Fiedorowicz H, Koshevoi M O, Rupasov A A, Rus B, Shikanov A S, Svoboda V 1991 X-ray transmission grating spectrometer with $\mathrm{CCD}$ detector for laser plasma studies. Laser Particle Beam 9: 579-591

Prasad Y B S R, Senecha V K, Vora H S, Khardekar R K, Pant H C 1998 Design and performance studies of an MCP-based pin-hole camera for laser-produced plasma diagnostics. Sadhana 23: 303-312

Radziemski L J, Cramers D A 1989 Laser induced plasma and application (New York: Marcel Dekker)

Rai V N 1998 Development of high speed plasma diagnostics and short puise laser plasma interaction. Contemporary science and technology of plasma (ed.) A K Gwal (New Delhi: Allied Publishers) pp 196-206

Rai V N, Shukla M 1994 A high voltage pulser circuit with subnanosecond risetime. Rev. Sci. Instrum. 65: 2134-2136

Rai V N, Shukla M, Desai T, Prasad Y B S R, Pant H C 1994a A detector for measurement of X-ray emission from laser produced plasmas. Plasma science and technology (ed.) I M L Das (New Delhi: Allied Publishers) pp 447-451

Rai V N, Shukla M. Pant H C 1994b A simple probe and attenuator for measurement of high voltage subnanosecond pulses. Meas. Sci. Technol. 5: 1396-1401

Rai V N, Desai T, Prasad Y B S R, Shukla M, Pant H C 1994c Laser ablation induced stress wave measurement in material. Proc. National Laser Symposium, CAT, Indore, pp 290-291

Rai V N, Shukla M, Khardekar R K 1994d A transistorised Marx bank circuit providing subnanosecond high voltage pulses. Meas. Sci. Technol. 5: 447-449

Rai V N. Shukla M, Pant H C, Bhawalkar D D 1995a Development of picosecond time resolution optical and X-ray streak cameras. Sādhanā 20: 937-954

Rai V N, Shukla M, Pant H C 1995b A multichannel vacuum photodiode and X-ray emission from a picosecond laser produced plasma. Proc. National Laser Symposium, Instrum. Res. Dev. Establishment, Dehradun, pp 269-270

Rai V N, Shukla M, Pant H C 1995c Generation of picosecond optical pulses from single hetrostructure GaAs diode laser and their emission characteristics. Pramana - J. Phys. 45: 439-451

Rai V N, Shukla M, Khardekar R K. Pant H C 1995d A picosecond optical pulse generator to calibrate the optical streak camera. Rev. Sci. Instrum. 66: 3125-3130

Rai V N, Shukla M, Pant H C 1996a Angle dependent X-ray emission from picosecond laser produced plasma. Recent advances in plasma science and technology (eds) R P Singh, S B Rai, D Narayan (New Delhi: Allied Publishers) 183-186

Rai V N, Shukla M, Pant H C 1996b Development of an intensifier based pin-hole camera and X-ray imaging of plasma expanding across the external magnetic field. Recent advances in plasma science and technology (eds) R P Singh, S B Rai, D Narayan (New Delhi: Allied Publishers) pp 191-194

Rai V N, Shukla M, Pant H C 1998a Some studies on picosecond laser produced plasma expanding across magnetic field. Laser Particle Beam 16: 431-443

Rai V N, Shukla M, Pant H C 1998b Effect of chamber pressure induced space charge potential on ion acceleration. Laser Particle Beam (in press)

Rai V N, Shukla M, Pant H C 1999 A biplanar vacuum photodiode and X-ray emission from magnetically confined laser produced plasma. Pramana - J. Phys. 52: 49-65

Ripin B H, McClean E A, Manka C K, Pawley C, Stamper J A, Peyser T A, Mostowych A N, Grun J, Hassam A B, Huba J 1987 Large larmor radius interchange instability. Phys. Rev. Lett. 59: 2299_ 2303

Salzmann D, Eliezer S, Krumbein A D, Gitter L 1983 Laser driven shock wave propagation in pure and layered targets. Phys. Rev. A28: 1738-1751

Schelev M Ya, Richardson M C, Alcock A J 1972 Operation of grid shuttered image converter tube in the picosecond region. Rev. Sci. Instrum. 43: 1819-1829

Schwanda W, Eidmann K 1992 Appl. Opt. 31: 554-559 
Shukla M, Rai V N, Pant H C 1996 A simple and compact high voltage switch mode power supply for streak camera. Meas. Sci. Technol. 7: 1668-1672

Shukla M, Rai V N, Pisharody S N, Pant H C 1997a A high speed biplanar vacuum photodiode for laser produced plasma. Proc. National Laser Symposium, CAT, Indore, pp 147-148

Shukla M, Rai V N, Pant H C. Bhawalkar D D 1997b Improvement in temporal resolution of optical and X-ray streak camera. National Symposium on Plasma Science and Technology, Institute of Plasma Research, Gandhinagar

Shukla M, Vitali, Chevokin V K, Rai V N, Pant H C 1998a A single frame framing camera. XIII National Symposium on Plasma Science and Technology, Saurastra University, Rajkot

Shukla M, Tripathi P K, Rai V N, Prasad Y B S R, Pant H C 1998b Development of X-ray transmission grating spectrometer for laser produced plasma. Contemporary science and technology of plasma (ed.) A K Gwal (New Delhi: Allied Publishers) pp 436-440

Shukla M, Chevokin V K, Rai V N, Pant H C 1998c A four frame framing camera for laser produced plasma. National Laser Symposium Indian Institute of Technology, Kanpur

Sigel R, Maaswinkel A G M, Tsakiris G D 1984 High speed diagnostics in laser fusion experiments. Proc. SPIE 491: 814-824

Sigel R, Eidmann K, Meyer-Ter-Vehn J, Tsakiris G D, Witkowski S 1987 Laser generated soft Xrays. Proc. SPIE 831: 73-83

Stearn D G, Wiedwald J D, Cook B M, Hanks R L, Landen O L 1989 X-ray framing camera for picosecond imaging of laser produced plasma. Rev. Sci. Instrum. 60: 363-367

Stradling G L, Attwood D T, Kauffman R L 1983 A soft X-ray streak camera. IEEE J. Quantum Electron. QE-19: 604-615

Tam A C 1986 Application of photoacoustic sensing technique. Rev. Mod. Phys. 58: 381-431

Tsuchiya Y 1983 Picosecond streak camera and its application. Picosecond, a technical bulletin of Hamamatsu 14: 2-18

Tsakiris G D 1989 A soft X-ray streak camera for laser plasma interaction studies. Proc. SPIE 1032: 910-931

Vora H S, Nakhe S V, Sarangapani K K, Saxena P, Bhatnagar R, Shirke N D 1994 Profile monitor for laser beam parameter measurements. Proc. National Laser Symposium, CAT, Indore, 260-261

Ze F, Kauffman R L, Kilkenny J D, Wielwald J, Bell P M, Hanks R, Stewarts J, Dean D, Bower J, Wallace R 1992 A new multichannel soft X-ray framing camera for fusion experiment. Rev. Sci. Instrum. 63: 5124-5126 